\title{
2D DOA estimation with sparse uniform circular arrays in the presence of mutual coupling
}

\author{
Julan Xie*, Zishu He, Huiyong Li and Jun Li
}

\begin{abstract}
In this article, we consider the uniform circular arrays (UCAs) with the number of antenna elements insufficient to apply the traditional beamspace-based algorithms, which are labeled as sparse UCAs. For such UCAs, we propose a new hybrid approach for 2D direction-of-arrival (DOA) estimation in the presence of mutual coupling. Using the manifold decomposition technique, we present two new formulations of the steering vector in the presence of mutual coupling for sparse UCAs. Then, we introduce the adaptations to a modified uniform circular array rank reduction algorithm. This leads to an algorithm that is able to estimate the azimuth angle without the exact knowledge of mutual coupling. Next, we use a search-free rooting algorithm which expands the steering into a double Fourier series for each estimated azimuth to obtain the elevation angle estimates. The manifold decomposition technique introduces truncation errors. However, the accuracy of the DOA estimates is strongly affected by these errors when the array has a small number of elements. Therefore, expressions describing the truncation errors in the DOA estimates are derived. This allows us to choose an appropriate truncated degree in the manifold separation transformation to enhance the DOA estimate accuracy. Numerical examples are presented to demonstrate the effectiveness of the proposed method.
\end{abstract}

Keywords: direction-of-arrival estimation, error analysis, manifold separation, rank reduction theory (RARE), sparse uniform circular array, wavefield modeling

\section{Introduction}

The problem of two-dimensional (2D) directions-of-arrival (DOAs) [1-7] estimation (i.e., azimuth and elevation angles) has received increasing attention in a variety of applications, such as radar, mobile communications, sonar, and seismology. In general, a planar array is needed when estimates of source azimuth and elevation are required. Such well-known planar arrays include the two-orthogonal uniform linear array (the L-shaped array) [1], the rectangular array [2], and the uniform circular array (UCA) [3-7]. The UCA is able to provide $360^{\circ}$ of coverage in the azimuth plane. Moreover, the UCA has uniform performance regardless of angle of arrival. Thus, UCA attracts more attention than other planar arrays recently. Due to the circular symmetry, the beamspace transformation, based on the phase-mode excitation principle, is usually applied to obtain the desired Vandermode structure for the steering vector in the mode space. This transformation results in

\footnotetext{
* Correspondence: xiezhu0415@yahoo.com.cn

School of Electronic Engineering, University of Electronic Science and Technology of China, Chengdu, Sichuan, 611731, China
}

the development of several DOA estimation algorithms with low computational cost, such as UCA-RB-MUSIC [3], UCA-ESPRIT [3], and uniform circular array rank reduction (UCA-RARE) [4]. However, all these algorithms for UCAs ignore the mutual coupling effect, which ultimately destroys the underlying model assumptions needed for their efficient implementations. Moreover, all these algorithms, based on the traditional beamspace transformation, require a sufficiently large number of elements to avoid aliasing in the steering vector of the mode space.

In this article, we focus on UCAs with the number of antenna elements insufficient to apply the traditional beamspace-based algorithms. In [8], such UCAs are labeled as sparse UCAs and are allowed to adopt an efficient search-free and robust 1D DOA estimation algorithm. This algorithm is based on a modified beamspace transformation and is called as sparse UCA RootMUSIC. In this algorithm, all relevant phased modes are able to be incorporated in a polynomial rooting procedure leading to biased free estimates when the number of elements of UCA is small. However, the algorithm in [8] 
only estimates the azimuth angle without considering the mutual coupling, given a fixed elevation angle. In this article, we estimate the 2D DOAs with such sparse UCAs in the presence of mutual coupling, acquiring both the azimuth and elevation angles estimates via a manifold decomposition technique. The straightforward extension of MUSIC to 2D DOA estimations brings on a 2D search over the MUSIC spectrum and has a high computational cost. In [5,7], both the proposed algorithms take the mutual coupling into account and employ the UCARARE algorithm to estimate the azimuth angle first. With the open-circuit voltages of the antenna elements expanded in spherical mode, a Root-MUSIC algorithm is able to be performed in the elevation space to obtain the elevation estimates in [5]. In [7], a 1D parameter search replaces the implementation of Root-MUSIC algorithm in the elevation space. In the 1D parameter search for elevation estimates, the elevation-dependent mutual coupling effect can efficiently be compensated by the elevation-dependent receiving mutual impedances. However, this step results in higher computational load. Although these two algorithms are applied for the compact UCAs, they could inspire us for sparse UCAs.

In this article, we use the method proposed in [9] to calculate the mutual coupling. In $[6,7,9]$, computer simulations have shown that this method can produce more accurate DOA estimation results than the open-circuit voltage method. The experiments in [6,7] show that the mutual coupling matrix (MCM) depends on the elevation angle for UCAs. Moreover, the simulation results in [6,7] have shown that the way compensating the mutual coupling with single-elevation-angle receiving-mutual-impedance, computed according to the method in [9], still produces better DOA estimation results than the opencircuit voltage method. In [7], it shows that the variation of the receiving mutual impedances with elevation angle is a process of gradual change. Hence, it is feasible to estimate the elevation angle using mutual coupling compensated with single-elevation-angle receiving-mutualimpedance. In [10], it is shown that any array steering vector can be expanded on a spherical surface to generate an expression containing spherical harmonics, which can be mapped to 2D Fourier basis [11]. In this article, we will extend this expansion to the steering vector in the presence of mutual coupling.

In this article, we propose a new hybrid algorithm for 2D DOA estimation in the presence of mutual coupling for sparse UCAs. Based on the manifold decomposition technique, we will present two new formulations of the steering vector in the presence of mutual coupling for sparse UCAs. One formulation, corresponding to JacobiAnger expansion [12], allows applying a modified UCARARE algorithm to estimate the azimuth angle without the exact knowledge of mutual coupling and elevation angle. The other formulation, corresponding to Bauer's formula [13], allows executing a Root-MUSIC algorithm in the elevation direction to estimate the elevation angle for each estimated azimuth angle. For sparse UCAs, compared with the original UCA-RARE, the modified UCARARE is able to avoid obtaining spurious estimates which only arise from the sparseness of the array elements. Note that the steering vector expansion for estimating the elevation angle in this article differs from that in [5] and has a more universal application $[10,14,15]$. In fact, these two kinds of decomposition techniques applied in this article can be considered as manifold decomposition transformations [11,16-18]. It is shown that the DOA estimate accuracy usually depends on the truncation error introduced by the transformation [16-19]. Hence, we analyze the truncation errors for sparse UCAs and derive expressions describing the truncation errors in the DOA estimates. We find that the impact of the truncation error on the estimate accuracy of azimuth angle is weaker than it for the elevation angle estimate. Therefore, a method to choose an appropriate truncated degree for the elevation estimates is presented to enhance the estimate accuracy.

The rest of the article is organized as follows. First, the array signal model is presented in Section 2, followed by the description of the manifold decomposition technique in Section 3. Then, the proposed algorithm for sparse UCA is presented in Section 4. The impact of the truncation errors are analyzed in Section 5. Section 6 shows the simulation results. Finally, Section 7 concludes the article.

\section{Array signal model}

Consider a sparse UCA consisting of $N$ identical elements uniformly distributed over the circumference of a circle of radius $r$. Assume that $D$ narrowband sources, centered on wavelength $\lambda$, impinge on the array from directions $\theta_{i}(i=1, \ldots, D)$ and $\varphi_{i}(i=1, \ldots, D)$, respectively, where $\theta_{i} \in[0, \pi / 2]$ is the elevation angle measured from the $Z$-axis and $\varphi_{i} \in[0,2 \pi)$ is the azimuth angle measured from the $X$-axis counter-clockwise (see Figure 1 ). The $N \times 1$ vector received by the array is expressed as

$$
\mathbf{x}(t)=\widetilde{\mathbf{A}}(\theta, \phi) \mathbf{s}(t)+\mathbf{n}(t)
$$

where $\widetilde{\mathbf{A}}(\theta, \phi)=\left[\widetilde{\mathbf{a}}\left(\theta_{1}, \phi_{1}\right) \ldots \widetilde{\mathbf{a}}\left(\theta_{D}, \phi_{D}\right)\right]$ is the $N \times$ $D$ matrix of the steering vectors, $\mathbf{s}(t)=\left[s_{1}(t) \ldots s_{D}(t)\right]^{T}$ is the $D \times 1$ signal vector, $\mathbf{n}(t)=\left[n_{1}(t) \ldots n_{N}(t)\right]^{T}$ is the $N \times 1$ noise vector. The signal vector $\mathbf{s}(t)$ and the vector $\mathbf{n}(t)$ of the additive and spatially white noise are assumed to be statistically independent and zero-mean.

If the sparse UCAs are composed of omni-directional antenna elements, its steering vector in the presence of mutual coupling [20] is given by 


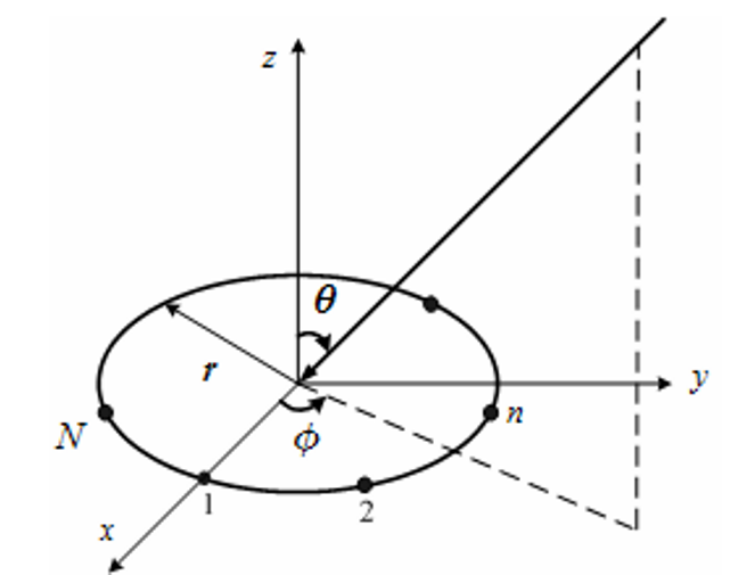

Figure 1 UCA deployed for direction finding

$$
\widetilde{\mathbf{a}}(\theta, \phi)=\mathbf{C}_{\mathrm{m}} \mathbf{a}(\theta, \phi)
$$

where the $N \times N$ matrix $\mathbf{c}_{\mathrm{m}}$ is the elevation-dependent MCM. Due to the circular symmetry, a model for the MCM of UCAs $[5,7,20]$ can be a complex symmetric circulant matrix. In this article, we only consider $N=$ $2 K+1$. Therefore, $\mathbf{C}_{\mathrm{m}}$ can be expressed as

$$
\mathbf{C}_{\mathrm{m}}=\left[\begin{array}{ccccccc}
c_{1} & c_{2} & \cdots & c_{(N+1) / 2} & c_{(N+1) / 2} & \cdots & c_{2} \\
c_{2} & c_{1} & \cdots & c_{(N+1) / 2-1} & c_{(N+1) / 2} & \cdots & c_{3} \\
\vdots & \vdots & \ddots & \vdots & \vdots & \ddots & \vdots \\
c_{2} & c_{3} & \cdots & c_{(N+1) / 2} & c_{(N+1) / 2-1} & \cdots & c_{1}
\end{array}\right]
$$

The $N \times 1$ vector $\mathbf{a}(\theta, \varphi)$ is the ideal steering vector and the expression of its $n$th $(n=1, \ldots, N)$ component is

$$
[\mathbf{a}(\theta, \phi)]_{n}=e^{j k r \sin \theta \cos \left(\phi-\gamma_{n}\right)}
$$

where $\gamma_{n}=2 \pi(n-1) / N$ is the angular position of the $n$th element.

If the sparse UCAs are composed of directional antenna elements, its steering vector in the presence of mutual coupling [21] can be described as

$$
\widetilde{\mathbf{a}}(\theta, \phi)=\mathbf{C}_{\mathrm{m}} \mathbf{a}_{\mathrm{d}}(\theta, \phi)
$$

where $\mathbf{a}_{\mathrm{d}}(\theta, \varphi)$ is the steering vector having the directional pattern of the form $g_{\mathrm{d}}(\theta, \varphi)$ and the expression of its $n$th $(n=1, \ldots N)$ component is

$$
\left[\mathbf{a}_{\mathrm{d}}(\theta, \phi)\right]_{n}=g_{\mathrm{d}}\left(\theta, \phi-\gamma_{n}\right) e^{j k r \sin \theta \cos \left(\phi-\gamma_{n}\right)}
$$

\section{The manifold decomposition technique}

Here, the concepts of the Wavefield modeling for scalarfields are given. Two expressions for decomposing the steering vector (manifold) of array elements with sparse UCAs are presented. These form the theoretical basis of our algorithm.
In [10], it is shown that the array steering vector is able to be decomposed as

$$
\mathbf{a}(\theta, \phi)=\boldsymbol{\Gamma}^{s} \mathbf{b}(\theta, \phi)
$$

where $\Gamma^{s}$ represents the so-called sampling matrix and $\mathbf{b}(\theta, \varphi)$ is the basis functions of the decomposition. In general, the dimension, i.e., the number of basis functions is infinite in order to hold the equality exactly. Therefore, the sampling matrix can be considered as an operator defined as $\Gamma^{s}: \mathcal{H} \rightarrow \mathbb{C}^{N \times 1}$. The coefficients of the expansion (sampling matrix) map functions defined on $\mathcal{H}$ into the $N$ th-dimensional complex space $\left(\mathbb{C}^{N \times 1}\right)$. Hence, the sampling matrix is a characteristic of the array only.

Usually there are two kinds of choices for the basis functions $\mathbf{b}(\theta, \varphi)$. For sparse UCA, one is given by

$$
[\mathbf{b}(\theta, \phi)]_{m}=j^{m} J_{m}(k r \sin \theta) e^{j m \phi}
$$

where $m=\ldots,-1,0,1, \ldots$ and $J_{m}(\bullet)$ is the Bessel function of the first kind with order $m$. Its corresponding sampling matrix is

$$
\left[\boldsymbol{\Gamma}^{s}\right]_{n, m}=e^{-j m \gamma_{m}}
$$

where $n=1,2, \ldots, N$. This kind of decomposition can also be considered as the Jacobi-Anger expansion [12], and Equation 4 can be expressed as

$$
e^{j k r \sin \theta \cos \left(\phi-\gamma_{n}\right)}=\sum_{m=-\infty}^{\infty} j^{m} J_{m}(k r \sin \theta) e^{j m\left(\phi-\gamma_{n}\right)}
$$

The other choice is the spherical harmonics and the th component of $\mathbf{b}(\theta, \varphi)$ is

$$
\begin{aligned}
{[\mathbf{b}(\theta, \phi)]_{t}=Y_{l}^{m}(\theta, \phi) } & =\sqrt{\frac{(2 l+1)(l-m) !}{4 \pi(l+m) !}} P_{l}^{m}(\cos \theta) e^{j m \phi} \\
l & \in[0,+\infty], m \in[-l, l]
\end{aligned}
$$

where $t=l^{2}+l+m+1$ and $P_{l}^{m}(\cos \theta)$ represents the associated Legendre functions of the $l$ th degree (or level) and $m$ th order (or mode). Its corresponding sampling matrix is

$$
\left[\Gamma^{s}\right]_{n, t}=4 \pi j^{l} j_{l}(k r) Y_{l}^{m *}\left(\frac{\pi}{2}, \gamma_{n}\right)
$$

where $j_{l}(\bullet)$ denotes the spherical Bessel function of the first kind. The Bauer's formula [13] corresponds to this kind of decomposition and is given by

$$
e^{j k r \sin \theta_{n} \cos \left(\phi-\gamma_{n}\right)}=4 \pi \sum_{l=0}^{\infty} \sum_{m=-l}^{l} j^{l} j_{l}(k r) Y_{l}^{m *}\left(\frac{\pi}{2}, \gamma_{n}\right) Y_{l}^{m}(\theta, \phi)
$$

Since both $J_{m}(k r)$ and $j_{l}(k r)$ decay exponentially, we can assume that, for $m \gg k r$ and $l \gg k r$, the higher- 
order Bessel functions and spherical Bessel functions are negligible. Therefore, the sampling matrix can be truncated by considering a finite number of modes or degrees. Ideally, the resulting truncation error can be made arbitrarily small just by increasing the number of modes or degrees. We assume that the truncated order is $M$ and truncated degree is $L$ for the first and second kinds of decomposition, respectively. The rule to select the truncated order or degree will be discussed in Section 5. In order to distinguish the sampling matrices and basis functions for two kinds of decomposition, let $\Gamma_{1}^{s}$ and $\mathbf{b}_{1}(\theta, \varphi)$ denote the sampling matrix and basis functions for the first kind of decomposition and $\boldsymbol{\Gamma}_{2}^{s}$ and $\mathbf{b}_{2}(\theta, \varphi)$ be the sampling matrix and basis functions for the second kind of decomposition, respectively.

In fact, an alternative expression [10] of $\mathbf{b}_{2}(\theta, \varphi)$ with limited degree $L$ is given by

$$
\mathbf{b}_{2}(\theta, \phi)=\mathbf{C}_{y} \mathbf{D}_{y}(\phi) \mathbf{Z}_{e} \mathbf{d}(\theta)
$$

where the $(L+1)^{2} \times(L+1)^{2}$ diagonal matrix $\mathbf{C}_{y}$ contains the diagonal elements $\left[\mathbf{C}_{\gamma}\right]_{t, t}=\sqrt{(2 l+1)(l-m) ! /(4 \pi(l+m) !)}$, $t=l^{2}+l+m+1 . \mathbf{D}_{y}(\phi) \in \mathbb{C}^{(L+1)^{2} \times(L+1)^{2}}$ is expressed as $\mathrm{Z}_{e} \in \mathbb{C}^{(L+1)^{2} \times(2 L+1)} \cdot \mathrm{Z}_{e} \in \mathbb{C}^{(L+1)^{2} \times(2 L+1)}$ is the combination of the selection matrix and the coefficients vectors and is given by $\mathbf{Z}_{e}=\left[\mathbf{Z}_{e}^{0 T} \mathbf{Z}_{e}^{1 T} \mathbf{Z}_{e}^{2 T} \ldots \mathbf{Z}_{e}^{L T}\right]^{T}$, where $\mathbf{Z}_{e}^{l}=\left[\begin{array}{llll}\mathbf{c}_{l}^{-l T} \cdots & \mathbf{c}_{l}^{0 T} \cdots \mathbf{c}_{l}^{l T}\end{array}\right]^{T} \in \mathbb{C}^{(2 l+1) \times(2 L+1)} \quad$ and $\widetilde{\mathbf{c}}_{l}^{m} \in \mathbb{C}^{(2 l+1) \times 1} . \widetilde{\mathbf{c}}_{l}^{m} \in \mathbb{C}^{(2 l+1) \times 1}$ can be obtained for an arbitrarily $l$ and $m$ using two recurrence expressions. The $(2 L+1) \times 1$ vector is described as $\mathbf{d}(\theta)=\left[\begin{array}{lllll}e^{-j L \theta} & \cdots & 1 & \cdots & e^{j L \theta}\end{array}\right]^{T}$. More details about Equation 14 can be found in [11]. Apparently, Equation 14 is an expansion of 2D Fourier series.

\section{The hybrid algorithm to DOA estimation}

For the ideal UCAs composed of omni-directional antenna elements, exciting the array with the weight vector $\mathbf{w}_{k}=1 / N\left[e^{-j k \gamma_{1}} \cdots e^{-j k \gamma_{N}}\right]^{H}$ results in

$$
\begin{aligned}
\mathbf{w}_{k}^{H} \mathbf{a}(\theta, \phi) & =j^{k} J_{k}(k r \sin \theta) e^{j k \phi}+ \\
& \sum_{q=1}^{\infty}\left[j^{k+q N} J_{k+q N}(k r \sin \theta) e^{j(k+q N) \phi}+\right. \\
& \left.j^{k-q N} J_{k-q N}(k r \sin \theta) e^{j(k-q N) \phi}\right]
\end{aligned}
$$

For the traditional beamspace transform, there is $N>2\lceil k r\rceil$ and the first term in (15) becomes dominant. However, for the beamspace transform applied to the UCA with $N=2 K+1$, where $K<\lceil k r\rceil$ and $N \leq 2\lceil k r\rceil$, the value of the second term may be significant and cannot be neglected because of the contribution of $J_{k \pm q N}(k r \sin \theta)$ with orders $K<|k| \leq\lceil k r\rceil$. We also label such UCAs as sparse UCAs [8]. Obviously, the algorithms proposed in [3-5,7], which are based on the traditional beamspace transform, cannot be employed directly for such UCAs. In this section, we will present a new hybrid algorithm applied to such UCAs. In order to avoid 2D search in MUSIC spectrum, we estimate the DOAs in two steps. Based on the beamspace transformation corresponding to the Jacobi-Anger expansion, first we estimate the azimuth angle using the modified UCA-RARE algorithm, which stems from the original UCA-RARE applied for compact UCA. This algorithm is attractive since it decouples azimuth estimation from elevation estimation and relaxes the assumption of omni-directional element patterns. Then, we perform a Root-MUSIC algorithm to estimate the elevation angle for every estimate azimuth angle using the expansion based on the Bauer's formula.

We start from the signal model of Section 2. Recalling Equation 1, the beamspace array signal model is

$$
\widetilde{\mathbf{x}}_{\mathbf{b}}(t)=\mathbf{W}_{K}^{H} \mathbf{x}(t)
$$

where the $(2 K+1) \times M$ weight matrix $\mathbf{W}_{k}$ is defined as

$$
\mathbf{W}_{K}=\sqrt{N}\left[\mathbf{w}_{-K} \ldots \mathbf{w}_{0} \ldots \mathbf{w}_{K}\right]
$$

The corresponding beamspace steering vector is

$$
\widetilde{\mathbf{a}}_{\mathrm{b}}(\theta, \phi)=\mathbf{W}_{K}^{H} \widetilde{\mathbf{a}}(\theta, \phi)
$$

The covariance matrix $\mathrm{R}$ of the beamspace data is constructed and an eigendecomposition of $\mathrm{R}$ results in a signal and noise subspace

$$
\mathbf{R}=E\left\{\widetilde{\mathbf{x}}_{\mathrm{b}}(t) \widetilde{\mathbf{x}}_{b}^{H}(t)\right\}=\widetilde{\mathbf{E}}_{\mathrm{s}} \widetilde{\Lambda}_{\mathrm{s}} \widetilde{\mathbf{E}}_{\mathrm{s}}^{H}+\widetilde{\mathbf{E}}_{\mathrm{n}} \widetilde{\Lambda}_{\mathrm{n}} \widetilde{\mathbf{E}}_{\mathrm{n}}^{H}
$$

where $\widetilde{\mathbf{E}}_{\mathrm{s}}$ and $\widetilde{\mathbf{E}}_{\mathrm{n}}$ denote the signal and noise subspace eigenvectors and the diagonal matrices $\widetilde{\Lambda}_{\mathrm{s}}$ and $\widetilde{\Lambda}_{\mathrm{n}}$ contain the signal subspace and noise subspace eigenvalues, respectively. The beamspace MUSIC algorithm estimates the DOAs from the $D$ deepest nulls of the MUSIC function

$$
f_{\text {MUSIC }}(\theta, \phi)=\widetilde{\mathbf{a}}_{\mathrm{b}}^{H}(\theta, \phi) \widetilde{\mathbf{E}}_{\mathrm{n}} \widetilde{\mathbf{E}}_{\mathrm{n}}^{H} \widetilde{\mathbf{a}}_{\mathrm{b}}(\theta, \phi)
$$

\subsection{The azimuth angle estimation}

When $\mathbf{a}(\theta, \varphi)$ is expanded as the first kind of decomposition, the beamspace steering vector $\mathbf{a}_{\mathrm{b}}(\theta, \varphi)$ can be expressed as

$$
\begin{aligned}
\mathbf{a}_{\mathrm{b}}(\theta, \phi) & =\mathbf{W}_{K}^{H} \boldsymbol{\Gamma}_{1}^{s} \mathbf{b}_{1}(\theta, \phi) \\
& =\mathbf{H T}(\phi) \mathbf{g}(\theta)+\Delta \mathbf{a}_{\mathrm{b}}^{1}(\theta, \phi)
\end{aligned}
$$


where

$$
\begin{aligned}
\mathbf{H}=\mathbf{W}_{K}^{H} \boldsymbol{\Gamma}_{1}^{s} & =\sqrt{N}\left[\mathbf{J}_{l} \mathbf{I}_{(2 K+1) \times(2 K+1)} \mathbf{J}_{r}\right] \in \mathbb{R}^{(2 K+1) \times(2 M+1)} \\
\mathbf{T}(\phi) & =\left[\begin{array}{cc}
\mathbf{Q}(z) & \mathbf{0}_{M \times 1} \\
\mathbf{0}_{1 \times M} & 1 \\
\mathbf{\Pi}(1 / z) & \mathbf{0}_{M \times 1}
\end{array}\right] \in \mathbb{C}^{(2 M+1) \times(M+1)} \\
\mathbf{Q}(z) & =\operatorname{diag}\left(z^{-M} z^{-M+1} \cdots z^{-2} z^{-1}\right) \\
z & =e^{j \phi} \\
{[\mathbf{g}(\theta)]_{m} } & =j^{M+1-m} J_{M+1-m}(k r \sin \theta), m=1,2, \cdots M+1
\end{aligned}
$$

The vector $\Delta \mathbf{a}_{\mathrm{b}}^{1}(\theta, \phi)$ represents the truncation errors term and its element is the summation of $j^{m} J_{m}(k r \sin \theta)$ $e^{j m \varphi}$ with $M<|m| \leq \infty$. This term can be arbitrarily small just by increasing $M$. The matrix $\mathbf{J}_{l}$ is a $(2 K+1) \times(M-K)$ matrix consisting of the $M-K$ last columns of the unity matrix $\mathbf{I}_{(2 K+1) \times(2 K+1)}$, whereas $\mathbf{J}_{r}$ is a $(2 K+1) \times(M-K)$ matrix consisting of the $M-K$ first columns of the unity matrix $\mathbf{I}_{(2 K+1) \times(2 K+1)}$. $\Pi$ is the $M \times M$ anti-diagonal matrix. Note that the expression of $\mathrm{H}$ in Equation 22 is restricted to $M<3 K+1$ [8]. The expression of Equation 21 is equivalent to the one for beamspace manifold in [8]. Now we will extend this transformation to the case considering the mutual coupling.

We should note that [22]

$$
\mathbf{C}_{\mathrm{m}}=\sum_{n=1}^{(N+1) / 2} c_{n} \mathbf{V}^{n}+\sum_{n=(N+1) / 2+1}^{N} c_{N+2-n} \mathbf{V}^{n}
$$

where $\mathbf{V}^{1}=\mathbf{I}$ and $\mathbf{V}^{n}(n>1)$ is the $(n-1)$ th power of the cyclic permutation operator given by

$$
\mathbf{V}=\left[\begin{array}{cc}
\mathbf{0}_{(N-1) \times 1} & \mathbf{I}_{(N-1) \times(N-1)} \\
1 & \mathbf{0}_{1 \times(N-1)}
\end{array}\right]
$$

Rewrite Equation 2 as

$$
\widetilde{\mathbf{a}}(\theta, \phi)=\sum_{n=1}^{(N+1) / 2} c_{n} \mathbf{V}^{n} \mathbf{a}(\theta, \phi)+\sum_{n=(N+1) / 2+1}^{N} c_{N+2-n} \mathbf{V}^{n} \mathbf{a}(\theta, \phi)
$$

Using $\mathbf{V}^{n} \mathbf{a}(\theta, \varphi)=\mathbf{a}(\theta, \varphi-2 \pi(n-1) / N)$ and Equation 21 yields

$$
\mathbf{W}_{K}^{H} \mathbf{V}^{n} \mathbf{a}(\theta, \phi)=\mathbf{H M}_{n} \mathbf{T}(\phi) \mathbf{g}(\theta)+\Delta \mathbf{a}_{\mathrm{b}}^{1}\left(\theta, \phi-\frac{2 \pi(n-1)}{N}\right)
$$

where

$\mathbf{M}_{n}=\operatorname{diag}\left\{e^{j 2 \pi K(n-1) / N} \cdots 1 \cdots e^{-j 2 \pi K(n-1) / N}\right\}$. Hence, Equation 18 can be re-expressed as

$$
\widetilde{\mathbf{a}}_{\mathrm{b}}(\theta, \phi)=\mathbf{H M}_{s} \mathbf{T}(\phi) \mathbf{g}(\theta)+\Delta \widetilde{\mathbf{a}}_{\mathrm{b}}^{1}(\theta, \phi)
$$

where $\quad \mathbf{M}_{s}=\sum_{n=1}^{(N+1) / 2} c_{n} \mathbf{M}_{n}+\sum_{n=(N+1) / 2+1}^{N} c_{N+2-n} \mathbf{M}_{n}$. The corresponding truncation errors term $\Delta \widetilde{\mathbf{a}}_{\mathrm{b}}^{1}(\theta, \phi)$ is expressed as

$$
\begin{aligned}
\Delta \widetilde{\mathbf{a}}_{\mathrm{b}}^{1}(\theta, \phi) & =\sum_{n=1}^{(N+1) / 2} c_{n} \Delta \mathbf{a}_{\mathrm{b}}^{1}\left(\theta, \phi-\frac{2 \pi(n-1)}{N}\right) \\
& +\sum_{n=(N+1) / 2+1}^{N} c_{N+2-n} \Delta \mathbf{a}_{\mathrm{b}}^{1}\left(\theta, \phi-\frac{2 \pi(n-1)}{N}\right)
\end{aligned}
$$

Because of the symmetry of the mutual coupling coefficients and the periodicity of $e^{j 2 \pi K(n-1) / N}$, it is easy to find that the diagonal elements of $\mathbf{M}_{s}$ is centro-symmetry. If we neglect the truncation errors term, Equation 27 can be modeled as

$$
\begin{aligned}
\widetilde{\mathbf{a}}_{\mathrm{b}}(\theta, \phi) & =\mathbf{H T}(\phi)(\mathbf{m} \odot \mathbf{g}(\theta)) \\
& =\mathbf{H T}(\phi) \widetilde{\mathbf{g}}(\theta)
\end{aligned}
$$

where $\tilde{\mathbf{g}}(\theta)=\mathbf{m} \odot \mathbf{g}(\theta)$ and $\mathbf{m}$ is the first $M+1$ elements of the diagonal elements of $\mathbf{M}_{s}$. “ $\odot$ " denotes the Hadamard product of vectors. The beamspace steering vector in the presence of mutual coupling for compact UCAs [5,7] is a special case of the one for sparse UCAs with $\mathbf{H}=\mathbf{I}$. Note that the components of $\widetilde{\mathbf{g}}(\theta)$ in Equation 29 have the same expression form with the ones in [7] and different expression form from the ones in [5].

Please also note that this transformation is able to be extended to the case that the sparse UCAs are composed of directional antenna elements. Recalling Equation 6 , there is

$$
\mathbf{w}_{k}^{H} \mathbf{a}_{\mathrm{d}}(\theta, \phi)=\frac{1}{N} \sum_{m=-\infty}^{+\infty} j^{m} J_{m}(k r \sin \theta) e^{j m \phi} \sum_{n=1}^{N} g_{\mathrm{d}}\left(\theta, \phi-\gamma_{n}\right) e^{j(k-m) \gamma_{n}}
$$

In general, the element directional pattern $g_{\mathrm{d}}(\theta, \varphi)$ [21] can be expressed as

$$
g_{\mathrm{d}}(\theta, \phi)=\sum_{p=-P}^{P} e_{p}(\theta) e^{j p \phi}
$$

Then, Equation 30 can be expressed as

$$
\mathbf{w}_{k}^{H} \mathbf{a}_{\mathrm{d}}(\theta, \phi)=\frac{1}{N} \sum_{p=-P}^{P} \sum_{m=-\infty}^{+\infty} j^{m} J_{m}(k r \sin \theta) e_{p}(\theta) e^{j(m+p) \phi} \sum_{n=1}^{N} e^{j(k-m-p) \gamma_{n}}
$$

It is easy to find that

$$
\sum_{n=1}^{N} e^{j(k-m-p) \gamma_{n}}= \begin{cases}N, & \text { if } m+p=k+q N, q \text { integer } \\ 0, & \text { otherwise }\end{cases}
$$

So that we finally get

$$
\begin{aligned}
& \mathbf{w}_{k}^{H} \mathbf{a}_{\mathrm{d}}(\theta, \phi)=v_{k}(\theta) e^{j k \phi}+ \\
& \quad \sum_{q=1}^{\infty}\left[v_{k+q N}(\theta) e^{j(k+q N) \phi}+v_{k-q N}(\theta) e^{j(k-q N) \phi}\right]
\end{aligned}
$$


where

$$
v_{k}(\theta)=\sum_{p=-P}^{P} j^{k-p} J_{k-p}(k r \sin \theta) e_{p}(\theta)
$$

Similarly, we can get the beamspace manifold in the presence of mutual coupling for arrays composed of directional elements

$$
\begin{aligned}
\widetilde{\mathbf{a}}_{\mathrm{b}}(\theta, \phi) & =\mathbf{H T}(\phi)(\mathbf{m} \odot \mathbf{g}(\theta)) \\
& =\mathbf{H T}(\phi) \widetilde{\mathbf{g}}(\theta)
\end{aligned}
$$

where $\widetilde{\mathbf{g}}(\theta)=\mathbf{m} \odot \mathbf{g}(\theta)$ and

$$
\begin{aligned}
{[\mathbf{g}(\theta)]_{m} } & =v_{M+1-m}(\theta) \\
& =\sum_{p=-P}^{P} j^{M+1-m-p} J_{M+1-m-p}(k r \sin \theta) e_{p}(\theta)
\end{aligned}
$$

Observing Equations 29 and 36, the beamspace manifolds for omni-directional elements and directional elements have the same expansion form with different components of $\mathbf{g}(\theta)$.

Replacing the beamspace steering vector by its factorization (29) or (36), the MUSIC function becomes

$$
f_{\text {MUSIC }}(\theta, \phi)=\widetilde{\mathbf{g}}^{H}(\theta) \mathbf{T}^{H}(\phi) \mathbf{H}^{H} \widetilde{\mathbf{E}}_{\mathbf{n}} \widetilde{\mathbf{E}}_{\mathrm{n}}^{H} \mathbf{H T}(\phi) \widetilde{\mathbf{g}}(\theta)
$$

When $N-D \geq M+1[4]$, this structure allows using a rank reduction algorithm, named UCA-RARE. Therefore, we can root the sample polynomial

$$
\left.P_{1}(z)\right|_{|z|=1}=\operatorname{det}\left\{\mathbf{T}^{T}(1 / z) \mathbf{H}^{H} \widetilde{\mathbf{E}}_{\mathbf{n}} \widetilde{\mathbf{E}}_{\mathbf{n}}^{H} \mathbf{H T}(z)\right\}
$$

and then find the signal azimuth angle from roots of (39), which are located closest to the unit circle. Note that $\operatorname{det}\{\bullet\}$ is the determinant of a matrix. Making use of a well-known identity for block matrices

$$
\begin{aligned}
\operatorname{det}\left\{\left[\begin{array}{ll}
\mathbf{A} & \mathbf{B} \\
\mathbf{C} & \mathbf{D}
\end{array}\right]\right\} & =\operatorname{det}\{\mathbf{A}\} \operatorname{det}\left\{\mathbf{D}-\mathbf{C A}^{-1} \mathbf{B}\right\} \\
& =\operatorname{det}\{\mathbf{D}\} \operatorname{det}\left\{\mathbf{A}-\mathbf{B D}^{-1} \mathbf{C}\right\}
\end{aligned}
$$

which holds true for arbitrary matrices $\mathbf{B}, \mathbf{C}$, and nonsingular matrices A, D. Using $\widetilde{\mathbf{E}}_{\mathbf{n}} \widetilde{\mathbf{E}}_{\mathrm{n}}^{H}=\mathbf{I}-\widetilde{\mathbf{E}}_{\mathrm{s}} \widetilde{\mathbf{E}}_{\mathrm{s}}^{H}$, Equation 39 becomes

$$
\begin{aligned}
\left.P_{1}(z)\right|_{|z|=1} & =\operatorname{det}\left\{\boldsymbol{\Psi}-\mathbf{T}^{T}(1 / z) \mathbf{H}^{H} \widetilde{\mathbf{E}}_{\mathrm{s}} \widetilde{\mathbf{E}}_{\mathrm{s}}^{H} \mathbf{H T}(z)\right\} \\
& =\operatorname{det}\left\{\left[\begin{array}{cc}
\mathbf{I} & \widetilde{\mathbf{E}}_{\mathrm{s}}^{H} \mathbf{H T}(z) \\
\mathbf{T}^{T}(1 / z) \mathbf{H}^{H} \widetilde{\mathbf{E}}_{\mathrm{s}} & \boldsymbol{\Psi}
\end{array}\right]\right\} \\
& =\operatorname{det}\{\boldsymbol{\Psi}\} \operatorname{det}\left\{\mathbf{I}-\widetilde{\mathbf{E}}_{\mathrm{s}}^{H} \mathbf{H T}(z) \boldsymbol{\Psi}^{-1} \mathbf{T}^{T}(1 / z) \mathbf{H}^{H} \widetilde{\mathbf{E}}_{\mathrm{s}}\right\}
\end{aligned}
$$

where $\boldsymbol{\Psi}=\mathbf{T}^{T}(1 / z) \mathbf{H}^{H} \mathbf{H T}(z)$. It is obvious that the roots of $\operatorname{det}\{\boldsymbol{\Psi}\}$, which are also the roots of (39), are only the spurious roots arising from the sparseness of the array elements and independent of the received data. Hence, a spatial spectrum function for the azimuth estimation with sparse UCAs can be constructed as

$$
\left.P_{2}(z)\right|_{|z|=1}=\frac{\operatorname{det}\left\{\mathbf{T}^{T}(1 / z) \mathbf{H}^{H} \widetilde{\mathbf{E}}_{\mathrm{n}} \widetilde{\mathbf{E}}_{\mathrm{n}}^{H} \mathbf{H T}(z)\right\}}{\operatorname{det}\{\boldsymbol{\Psi}\}}
$$

or

$$
\left.P_{2}(z)\right|_{|z|=1}=\operatorname{det}\left\{\mathbf{I}-\tilde{\mathbf{E}}_{s}^{H} \mathbf{H T}(z) \Psi^{-1} \mathbf{T}^{T}(1 / z) \mathbf{H}^{H} \tilde{\mathbf{E}}_{s}\right\}
$$

However, there may be common roots for $\operatorname{det}\{\boldsymbol{\Psi}\}$ and $\operatorname{det}\left\{\mathbf{I}-\widetilde{\mathbf{E}}_{\mathrm{s}}^{H} \mathbf{H T}(z) \boldsymbol{\Psi}^{-1} \mathbf{T}^{T}(1 / z) \mathbf{H}^{H} \widetilde{\mathbf{E}}_{\mathrm{s}}\right\}$, which may be the true roots. In order to avoid eliminating such roots, it is better to use Equation 43 to acquire the azimuth estimate. Notice that the original UCA-RARE algorithm, based on the traditional beamspace transform, is a special case of the modified UCA-RARE algorithm in this study. For traditional beamspace transform, there is $\mathbf{H}=$ I and $\operatorname{det}\{\boldsymbol{\Psi}\}=2^{M}$. Hence, the roots of Equation 43 are equivalent to these of Equation 39 for original UCARARE algorithm.

Similar to the Root-MUSIC roots, RARE roots enjoy the so-called conjugate reciprocity property, i.e., if $z_{0}$ is a root of $P_{2}(z)$, then $\tilde{z}_{0}=1 / z_{0}^{*}$ is also a root of $P_{2}(z)$. Therefore, there are spurious estimates $\varphi_{i}+\pi$ for $\varphi_{i}<\pi$ and $\varphi_{i}-\pi$ for $\varphi_{i}>\pi$. Although there are still spurious estimates $\left(\varphi_{\mathrm{i}}+\varphi_{j}\right) / 2$ for the case of impinging sources with the same elevation angle $\left(\theta_{i}=\theta_{j}\right)$, we do not plan to eliminate them in order to avoid cancelling the real root at $\left(\varphi_{\mathrm{i}}+\varphi_{j}\right) / 2$ when there is a source exactly at $\left(\varphi_{\mathrm{i}}+\varphi_{j}\right) / 2$. Besides all these spurious estimates, there may be other spurious estimates introduced by the sparseness of the array elements. However, all spurious estimates can be eliminated in the final paired 2D DOA estimation by the elevation estimate in the next step.

\subsection{The elevation angle estimation}

A specifically designed closed-form algorithm similar to UCA-ESPRIT is proposed in the original UCA-RARE algorithm [4] to obtain the elevation estimates. Although it is a search-free implementation, there are some shortcomings that make it somewhat unsuitable for practical application, which are presented in detail in [7]. Hence, we apply for the Root-MUSIC algorithm via decomposing the steering vector into the double Fourier series to estimate the elevation angle. Note that the steering vector expansion in the presence of mutual coupling for estimating the elevation angle in this article differs from that in [5]. The method in [5], which estimates the DOAs for compact UCAs, is based on the open-circuit voltages of the antenna elements expanded in spherical mode, whereas our 
method, in which the mutual coupling is calculated by the proposed approach in [9], grounds on the manifold decomposition. In [5], the steering vector is expanded into a limited Fourier series of phase modes by considering a general multiport antenna, carrying a current distribution $\mathbf{C}(r, \phi, z)$ on the surface $S$ of a cylinder with radius $r$ and height $z_{\max }$. In our proposed method, the steering vector is expanded into a limited Fourier series of phase modes by considering an element on the surface of a unit sphere. Moreover, the truncation degree in [5] is determined by the radius $r$ and height $z_{\max }$ together, while the one in our proposed algorithm is only relative to $r$.

In [7], it shows that the variation of the receiving mutual impedances with elevation angle is a process of gradual change. It means that the receiving mutual impedances do not vary with elevation angle significantly. The simulation results in $[6,7]$ have shown that estimating the elevation angle with single-elevation-angle receiving-mutual-impedance could achieve error accuracy around $1^{\circ}$. Therefore, it is feasible to estimate the elevation angle using mutual coupling compensated with single-elevation-angle receiving-mutual-impedance. We will estimate an initial elevation angle using the MCM obtained at $\theta=45^{\circ}$ first. Then we can get a more accurate result with the MCM obtained at the initial estimate.

When $\mathbf{a}(\theta, \varphi)$ is expanded as the second kind of decomposition and $\mathbf{b}_{2}(\theta, \varphi)$ is decomposed as Equation 14 , the beamspace steering vector $\mathbf{a}_{\mathrm{b}}(\theta, \varphi)$ can be expressed as

$$
\begin{aligned}
\mathbf{a}_{\mathrm{b}}(\theta, \phi) & =\mathbf{W}_{K}^{H} \boldsymbol{\Gamma}_{2}^{s} \mathbf{b}_{2}(\theta, \phi) \\
& =\mathbf{B C}_{\gamma} \mathbf{D}_{\gamma}(\phi) \mathbf{Z}_{e} \mathbf{d}(\theta)+\Delta \mathbf{a}_{\mathrm{b}}^{2}(\theta, \phi)
\end{aligned}
$$

where $\mathbf{B}=\mathbf{W}_{K}^{H} \boldsymbol{\Gamma}_{2}^{s}$ and $\Delta \mathbf{a}_{\mathrm{b}}^{2}(\theta, \phi)$ represent the truncation errors term of the second kind of decomposition. The $m$ th elements of $\Delta \mathbf{a}_{b}^{2}(\theta, \phi)$ is

$$
\left[\Delta \mathbf{a}_{\mathrm{b}}^{2}(\theta, \phi)\right]_{n}=\frac{4 \pi}{N} \sum_{l=L+1}^{\infty} \sum_{m=-l}^{l} j^{l} j_{l}(k r) Y_{l}^{m}(\theta, \phi) \sum_{n=1}^{N} e^{j t \gamma_{n}} Y_{l}^{m *}\left(\frac{\pi}{2}, \gamma_{n}\right)
$$

where $t=K+1-n$. In the presence of mutual coupling, the corresponding beamspace steering vector $\widetilde{\mathbf{a}}_{\mathbf{b}}(\theta, \phi)$, defined in Equation 18, is

$$
\begin{aligned}
\widetilde{\mathbf{a}}_{\mathrm{b}}(\theta, \phi) & =\mathbf{W}_{K}^{H} \mathbf{C}_{m} \boldsymbol{\Gamma}_{2}^{s} \mathbf{b}_{2}(\theta, \phi) \\
& =\widetilde{\mathbf{B}} \mathbf{C}_{\gamma} \mathbf{D}_{\gamma}(\phi) \mathbf{Z}_{e} \mathbf{d}(\theta)+\Delta \widetilde{\mathbf{a}}_{\mathrm{b}}^{2}(\theta, \phi)
\end{aligned}
$$

where $\widetilde{\mathbf{B}}=\mathbf{W}_{K}^{H} \mathbf{C}_{m} \boldsymbol{\Gamma}_{2}^{s}$ and $\Delta \widetilde{\mathbf{a}}_{\mathrm{b}}^{2}(\theta, \phi)$ represent the corresponding truncation errors term. The $n$th elements of $\Delta \widetilde{\mathbf{a}}_{\mathrm{b}}^{2}(\theta, \phi)$ is

$$
\begin{aligned}
& {\left[\Delta \widetilde{\mathbf{a}}_{\mathrm{b}}^{2}(\theta, \phi)\right]_{n}=\frac{4 \pi}{N} \sum_{l=L+1}^{\infty} \sum_{m=-l}^{l} j^{l} j_{l}(k r) Y_{l}^{m}(\theta, \phi)} \\
& \quad \cdot\left(\sum_{q=1}^{(N+1) / 2} c_{q} e^{j t \gamma_{n}} Y_{l}^{m *}\left(\frac{\pi}{2}, \gamma_{n}\right)+\sum_{q=(N+1) / 2+1}^{N} c_{N+2-q} e^{j t \gamma_{n}} Y_{l}^{m *}\left(\frac{\pi}{2}, \gamma_{n}\right)\right)
\end{aligned}
$$

If neglect the truncation errors term $\Delta \widetilde{\mathbf{a}}_{\mathrm{b}}^{2}(\theta, \phi)$, the beamspace MUSIC function becomes

$$
f_{\text {MUSIC }}(\theta, \phi)=\mathbf{d}^{H}(\theta) \mathbf{Z}_{e}^{H} \mathbf{D}_{y}^{H}(\phi) \mathbf{C}_{y}^{H} \widetilde{\mathbf{B}}^{H} \widetilde{\mathbf{E}}_{\mathrm{n}} \widetilde{\mathbf{E}}_{\mathrm{n}}^{H} \widetilde{\mathbf{B}} \mathbf{C}_{y} \mathbf{D}_{y}(\phi) \mathbf{Z}_{e} \mathbf{d}(\theta)
$$

Apparently, this equation is a polynomial in $w=e^{j \theta}$ for each estimate azimuth angle $\varphi_{i}$. The Root-MUSIC algorithm can be performed to obtain the elevation angle estimates. A method, which extends the steering vector in the elevation field from $[0, \pi]$ to $[0,2 \pi]$, is presented in $[5]$ to decrease the implementation times of the Root-MUSIC algorithm. In fact, this method only considers the case that there are only spurious estimate $\varphi_{i}+\pi$. This will result in errors for $\varphi_{i}>\pi$ whose spurious estimate is $\varphi_{i}-\pi$. Hence, we prefer to perform Root-MUSIC algorithm for each azimuth estimates separately rather than perform Root-MUSIC algorithm with combining $\varphi_{i}$ and $\varphi_{i}+\pi$. Note that there are usually two optimal solutions $\theta_{i}$ and $\pi$ - $\theta_{i}$ for one azimuth angle $\varphi_{i}$ due to $\sin \theta_{i}=\sin \left(\pi-\theta_{i}\right)$. This characteristic is determined by the symmetry of the circular array's manifold in the elevation range. The real root should be located at $(0, \pi / 2]$ for a circular array. It is clear that the following equation holds true for $(\theta, \varphi)$ :

$$
\begin{aligned}
\mathbf{b}_{2}(\theta, \phi) & =\mathbf{C}_{\gamma} \mathbf{D}_{y}(\phi) \mathbf{Z}_{e} \mathbf{d}(\theta) \\
& =\mathbf{C}_{\gamma} \mathbf{D}_{\gamma}(\phi+\pi) \mathbf{Z}_{e} \mathbf{d}(2 \pi-\theta) \\
& =\mathbf{C}_{\gamma} \mathbf{D}_{\gamma}(\phi-\pi) \mathbf{Z}_{e} \mathbf{d}(2 \pi-\theta)
\end{aligned}
$$

That shows that the solutions to $\varphi_{i}+\pi$ or $\varphi_{i}-\pi$ are $2 \pi-\theta \in[3 \pi / 2,2 \pi]$ and $2 \pi-(\pi-\theta)=\pi+\theta \in[\pi, 3 \pi / 2]$. So, this algorithm allows eliminating the spurious estimate $\varphi_{i}+\pi$ or $\varphi_{i}-\pi$ automatically. Again the spurious estimates $\left(\varphi_{\mathrm{i}}+\varphi_{j}\right) / 2$ and $\left(\varphi_{\mathrm{i}}+\varphi_{j}\right) / 2+\pi$ can only keep one result if there are sources with the same elevation angle $\left(\theta_{i}=\theta_{j}\right)$. Although all spurious azimuth estimates are considered, we only reserve the paired DOAs $\left(\theta_{i}, \varphi_{i}\right)$ whose elevation estimates locate at $[0, \pi / 2]$. The number of such paired estimate $\left(\theta_{i}, \varphi_{i}\right)$ may be more than $D$. Hereby, it is necessary to calculate the MUSIC function for every paired estimate $\left(\theta_{i}, \varphi_{i}\right)$. Only the $D$ smallest values of the MUSIC function are considered as the final estimates for the DOAs $(\theta, \varphi)$.

For the sparse UCAs composed of directional antenna elements, we could still execute the Root-MUSIC algorithm. Recalling Equations 6 and 13, there is

$$
\begin{aligned}
{\left[\mathbf{a}_{\mathrm{d}}(\theta, \phi)\right]_{n} } & =g_{\mathrm{d}}\left(\theta, \phi-\gamma_{n}\right) e^{j k r \sin \theta \cos \left(\phi-\gamma_{n}\right)} \\
& =4 \pi g_{\mathrm{d}}\left(\theta, \phi-\gamma_{n}\right) \\
& \cdot \sum_{l=0}^{\infty} \sum_{m=-l}^{l} j^{l} j_{l}(k r) Y_{l}^{m *}\left(\frac{\pi}{2}, \gamma_{n}\right) Y_{l}^{m}(\theta, \phi)
\end{aligned}
$$

Then, the components of the sampling matrix becomes

$$
\left[\Gamma^{s}\right]_{n, t}=4 \pi j^{l} j_{l}(k r) g_{\mathrm{d}}\left(\theta, \phi-\gamma_{n}\right) Y_{l}^{m *}\left(\frac{\pi}{2}, \gamma_{n}\right)
$$


Since the value of the azimuth angle has been estimated, $g_{\mathrm{d}}\left(\theta, \varphi-\gamma_{n}\right)$ is only a function of elevation angle $\theta$ and can be labeled as $g_{\mathrm{d}}^{n}(\theta)$. Usually the direction pattern $g_{\mathrm{d}}(\theta)$ is able to be expressed as a function of $\cos \theta$ and $\sin \theta$. We define $w=e^{j \theta}$. It is easy to get $\cos \theta=(w$ $\left.+w^{-1}\right) / 2$ and $\sin \theta=-j\left(w-w^{-1}\right) / 2$. In such case, the beamspace steering vector $\widetilde{\mathbf{a}}_{\mathrm{b}}(\theta, \phi)$ has the same expansion form as it is in Equation 46 but with different components of the sampling matrix. It could sill be written as a polynomial in $w$. Hence, the Root-MUSIC algorithm is still able to be performed to estimate the elevation angle.

The steps involved in the proposed hybrid algorithm can be summarized below:

(1) Compute the sample covariance matrix $\widehat{\mathbf{R}}=(1 / P) \sum_{p=1}^{P} \mathbf{x}(p) \mathbf{x}^{H}(p)$ by averaging over $P$ data snapshots. Compute the beamspace covariance matrix $\widehat{\mathbf{R}}_{b}=\mathbf{W}_{K}^{H} \widehat{\mathbf{R}} \mathbf{W}_{K}$.

(2) Perform the eigenvalue decomposition of $\widehat{\mathbf{R}}_{b}$. Form the matrix $\widehat{\mathbf{E}}_{s}$ and $\widehat{\mathbf{E}}_{n}$, which spans the estimated signal subspace and the noise subspace, respectively.

(3) Obtain the azimuth angle estimates with Equation 43. All spurious azimuth estimates are reserved.

(4) For every reserved azimuth estimate, perform the Root-MUSIC with the MCM obtained at $\theta=45^{\circ}$ to find an initial elevation estimates. Since the location of the real elevation angle is $\theta \in(0, \pi / 2]$, except for the case that there is a source exactly at $\varphi_{i}+\pi$ or $\varphi_{i}-\pi$, all spurious estimates $\varphi_{i}+\pi$ or $\varphi_{i}-\pi$ can be eliminated automatically (see Equation 49). Then, we perform the Root-MUSIC with the MCM obtained at initial estimate to get a more accurate estimate.

(5) Calculate the MUSIC function for every paired estimate $\left(\theta_{i}, \varphi_{i}\right)$. Take the paired estimates $(\theta, \varphi)$ corresponding to the $D$ smallest values of the MUSIC function as the final estimate.

\section{The impact of the truncation errors on the estimation accuracy}

As discussed in Sections 3 and 4, the manifold decomposition will introduce truncation errors. Here, a firstorder approximation of the bias based on manifold decomposition is derived for sparse UCAs, and a thumb rule to choose the truncate degree is presented.

The truncation errors terms $\Delta \widetilde{\mathbf{a}}_{\mathrm{b}}^{i}(\theta, \phi)(i=1,2)$ in Equations 28 and 47 are dependent on the DOAs $(\theta, \varphi)$ and truncation degree $L$ or order $M$. Let's consider a sparse UCA with $N=11$ monopoles tuned to $f_{0}=2.4$ GHz. The radius is $r=\lambda$. The monopole elements are of equal length $3.13 \mathrm{~cm}$ and radius is $0.3 \mathrm{~mm}$. All monopole elements are loaded with a terminal load $Z_{0}$ $=50 \Omega$. The receiving mutual impedances shown in Table 1 are calculated with receiving-mutual-impedance method [9] for different elevation angles of an impinging source. Figure 2 shows the value of $\left\|\Delta \widetilde{\mathbf{a}}_{\mathrm{b}}^{i}(\theta, \phi)\right\|(i=1$, 2) as a function of both azimuth angle $\varphi$ and elevation angle $\theta$ for this UCA with $L=M=\lceil k r\rceil=7$. Notice that it needs more than 15 elements for the traditional beamspace transform. It is easy to see that $\left\|\Delta \widetilde{\mathbf{a}}_{\mathrm{b}}^{1}(\theta, \phi)\right\|$ is always smaller than $\left\|\Delta \widetilde{\mathbf{a}}_{\mathrm{b}}^{2}(\theta, \phi)\right\|$ when these two kinds of decomposition are performed with the same DOAs $(\theta, \varphi)$ and truncation degree $L$ or order $M$. $\left\|\Delta \widetilde{\mathbf{a}}_{\mathrm{b}}^{1}(\theta, \phi)\right\|$ decreases as the elevation angle changes from $90^{\circ}$, while $\left\|\Delta \widetilde{\mathbf{a}}_{\mathrm{b}}^{2}(\theta, \phi)\right\|$ varies over the elevation angle and hold bigger for the angle near $0^{\circ}$ and $90^{\circ}$. Both $\left\|\Delta \widetilde{\mathbf{a}}_{\mathrm{b}}^{1}(\theta, \phi)\right\|$ and $\left\|\Delta \widetilde{\mathbf{a}}_{\mathrm{b}}^{2}(\theta, \phi)\right\|$ nearly have the same value over the azimuth angle for a fixed elevation angle.

\subsection{Analysis of the bias in the azimuth angle estimation} As we know, we estimate the azimuth angle based on the rank reduction theory. For the true $\varphi_{i}$, there is

$$
\begin{aligned}
0 & =P_{2}\left(\phi_{i}\right)=P_{1}\left(\phi_{i}\right) \\
& =\operatorname{det}\left\{\mathbf{I}-\widetilde{\mathbf{E}}_{\mathrm{s}}^{H} \mathbf{H T}\left(\phi_{i}\right) \boldsymbol{\Psi}^{-1} \mathbf{T}^{H}\left(\phi_{i}\right) \mathbf{H}^{H} \widetilde{\mathbf{E}}_{\mathrm{s}}\right\} \\
& =\operatorname{det}\left\{\mathbf{T}^{H}\left(\phi_{i}\right) \mathbf{H}^{H} \widetilde{\mathbf{E}}_{\mathbf{n}} \widetilde{\mathbf{E}}_{\mathbf{n}}^{H} \mathbf{H T}\left(\phi_{i}\right)\right\}
\end{aligned}
$$

Let $\mathbf{F}\left(\phi_{i}\right)=\mathbf{T}^{H}\left(\phi_{i}\right) \mathbf{H}^{H} \widetilde{\mathbf{E}}_{\mathbf{n}} \widetilde{\mathbf{E}}_{\mathbf{n}}^{H} \mathbf{H T}\left(\phi_{i}\right)$ and the eigendecomposition of $\mathbf{F}\left(\varphi_{i}\right)$ is given by

$$
\mathbf{F}\left(\phi_{i}\right)=\mathbf{U}_{\mathrm{s}} \boldsymbol{\Sigma}_{s} \mathbf{U}_{s}^{H}+\mathbf{U}_{\mathrm{n}} \boldsymbol{\Sigma}_{\mathrm{n}} \mathbf{U}_{\mathrm{n}}^{H}
$$

If there are $m_{i}$ sources with the same azimuth angle $\varphi_{i}$ and $n_{i}$ sources with the azimuth angle $\varphi_{i}+\pi$ or $\varphi_{i}-\pi$, then $\Sigma_{s}$ denotes the diagonal matrix containing the D$\left(m_{i}+n_{i}\right)$ non-zero eigenvalues and $\boldsymbol{\Sigma}_{\mathrm{n}}$ contains the remaining $m_{i}+n_{i}$ zero eigenvalues [4,7]. The matrices $\mathbf{U}_{s}$ and $\mathbf{U}_{\mathrm{n}}$ in turn contain the corresponding eigenvectors, respectively. Define a function

$$
\begin{aligned}
y\left(\phi_{i}\right) & =\sum_{q=1}^{m_{i}+n_{i}} \mathbf{u}_{q}^{H} \mathbf{F}\left(\phi_{i}\right) \mathbf{u}_{q} \\
& =\sum_{q=1}^{m_{i}+n_{i}} \mathbf{u}_{q}^{H} \mathbf{T}^{H}\left(\phi_{i}\right) \mathbf{H}^{H} \widetilde{\mathbf{E}}_{\mathbf{n}} \widetilde{\mathbf{E}}_{\mathbf{n}}^{H} \mathbf{H T}\left(\phi_{i}\right) \mathbf{u}_{q}
\end{aligned}
$$

where $\mathbf{u}_{q}$ represents the $q$ th columns of $\mathbf{U}_{\mathrm{n}}$. Due to the truncation errors, $y\left(\varphi_{i}\right) \neq 0$ but $y\left(\varphi_{i}\right) \approx 0$. An expression for the basis can be found by expanding the first derivative of Equation 54 with respect to $\varphi_{i}$ and 
Table 1 The receiving mutual impendence at different elevation angles of impinging sources

\begin{tabular}{llllll}
\hline Elevation & $Z_{12}$ & $Z_{13}$ & $Z_{14}$ & $Z_{15}$ & $Z_{16}$ \\
\hline $10^{\circ}$ & $i^{*} 11.7873+4.5314$ & $-2.4943-i^{*} 8.9404$ & $i^{*} 6.3951+1.4430$ & $-i^{*} 4.3664-1.1790$ & $i^{*} 3.3245+0.7546$ \\
$20^{\circ}$ & $i^{*} 11.8023+4.4814$ & $-2.5015-i^{*} 8.9513$ & $i^{*} 6.3998+1.4395$ & $-i^{*} 4.3638-1.1815$ & $i^{*} 3.2703+0.7552$ \\
$30^{\circ}$ & $i^{*} 11.8237+4.4157$ & $-2.5233-i^{*} 8.9649$ & $i^{*} 6.4139+1.4288$ & $-i^{*} 4.3570-1.1989$ & $i^{*} 3.2342+0.7563$ \\
$40^{\circ}$ & $i^{*} 11.8401+4.3538$ & $-2.5602-i^{*} 8.9711$ & $i^{*} 6.4369+1.4101$ & $-i^{*} 4.3487-1.2203$ & $i^{*} 3.2053+0.7578$ \\
$50^{\circ}$ & $i^{*} 11.8519+4.3054$ & $-2.6118-i^{*} 8.9714$ & $i^{*} 6.4659+1.3819$ & $-i^{*} 4.3375-1.2510$ & $i^{*} 3.1950+0.7586$ \\
$60^{\circ}$ & $i^{*} 11.8583+4.2649$ & $-2.6728-i^{*} 8.9617$ & $i^{*} 6.4929+1.3353$ & $-i^{*} 4.3190-1.3035$ & $i^{*} 3.1834+0.7595$ \\
$70^{\circ}$ & $i^{*} 11.8629+4.2227$ & $-2.7281-i^{*} 8.9483$ & $i^{*} 6.5087+1.3001$ & $-i^{*} 4.3032-1.3261$ & $i^{*} 3.1755+0.7609$ \\
$80^{\circ}$ & $i^{*} 11.8663+4.1837$ & $-2.7606-i^{*} 8.9472$ & $i^{*} 6.5152+1.2800$ & $-i^{*} 4.2933-1.3392$ & $i^{*} 3.1676+0.7618$ \\
$90^{\circ}$ & $i^{*} 11.8654+4.1721$ & $-2.7682-i^{*} 8.9458$ & $i^{*} 6.5202+1.2780$ & $-i^{*} 4.2900-1.3435$ & $i^{*} 3.1557+0.7621$ \\
\hline
\end{tabular}

evaluating at $\hat{\phi}_{i}$. For small enough errors, we have $[16,17,23]$ :

$$
0=\gamma^{\prime}\left(\hat{\phi}_{i}\right) \approx \gamma^{\prime}\left(\phi_{i}\right)+\gamma^{\prime \prime}\left(\phi_{i}\right)\left(\hat{\phi}_{i}-\phi_{i}\right)
$$

where $\hat{\phi}_{i}$ and $\varphi_{i}$ are the estimated and true azimuth angle and $\left.\gamma^{\prime}\left(\hat{\phi}_{i}\right) \triangleq(\partial y(\phi) / \partial \phi)\right|_{\phi=\hat{\phi}_{i}}$. Let $\mathbf{T}^{\prime}(\varphi) \triangleq \partial \mathbf{T}$ $(\varphi) / \partial \varphi$ and, similarly, $\mathbf{T}^{\prime \prime}(\varphi) \triangleq \partial \mathbf{T}^{\prime}(\varphi) / \partial \varphi$. The first derivative of $y(\varphi)$ is

$$
\gamma^{\prime}(\phi)=\sum_{q=1}^{m_{i}+n_{i}} 2 \operatorname{Re}\left\{\mathbf{u}_{q}^{H} \mathbf{T}^{H}(\phi) \mathbf{H}^{H} \widetilde{\mathbf{E}}_{\mathbf{n}} \widetilde{\mathbf{E}}_{\mathbf{n}}^{H} \mathbf{H} \mathbf{T}^{\prime}(\phi) \mathbf{u}_{q}\right\}
$$

where $\operatorname{Re}\{\cdot\}$ stands for the real part of the argument within the brackets. Similarly, the second derivative of $y$ $(\varphi)$ is yielded as

$$
\begin{aligned}
y^{\prime \prime}(\phi) & =\frac{\partial y^{\prime}(\phi)}{\partial \phi} \\
& =\sum_{q=1}^{m_{i}+n_{i}} 2 \mathbf{u}_{q}^{H} \mathbf{T}^{H}(\phi) \mathbf{H}^{H} \widetilde{\mathbf{E}}_{\mathbf{n}} \widetilde{\mathbf{E}}_{\mathbf{n}}^{H} \mathbf{H T}^{\prime}(\phi) \mathbf{u}_{q} \\
& +\sum_{q=1}^{m_{i}+n_{i}} 2 \operatorname{Re}\left\{\mathbf{u}_{q}^{H} \mathbf{T}^{H}(\phi) \mathbf{H}^{H} \widetilde{\mathbf{E}}_{\mathrm{n}} \widetilde{\mathbf{E}}_{\mathbf{n}}^{H} \mathbf{H T}^{\prime \prime}(\phi) \mathbf{u}_{q}\right\}
\end{aligned}
$$

By combining (55)-(57), the bias for the azimuth angle estimates at angle $\varphi_{i}$ can finally be computed from

$$
\hat{\phi}_{i}-\phi_{i} \approx-\frac{y^{\prime}\left(\phi_{i}\right)}{\gamma^{\prime \prime}\left(\phi_{i}\right)}
$$

5.2. Analysis of the bias in the elevation angle estimation Similarly, an expression for the elevation estimation basis can be found by expanding the first derivative of Equation 48 with respect to $\theta_{i}$ and evaluating at $\hat{\theta}_{i}$. For small enough errors, there is $[16,17,23]$ :

$$
0=f^{\prime}\left(\hat{\theta}_{i}\right) \approx f^{\prime}\left(\theta_{i}\right)+f^{\prime \prime}\left(\theta_{i}\right)\left(\hat{\theta}_{i}-\theta_{i}\right)
$$

where $\hat{\theta}_{i}$ and $\theta_{i}$ are the estimated and true elevation angle and $\left.f^{\prime}\left(\hat{\theta}_{i}\right) \triangleq(\partial f(\theta) / \partial \theta)\right|_{\theta=\hat{\theta}_{i}}$. We define the vectors $\mathbf{d}^{\prime}(\theta)=\partial \mathbf{d}(\theta) / \partial \theta$ and $\mathbf{d}^{\prime \prime}(\theta)=\partial \mathbf{d}^{\prime}(\theta) / \partial \theta$. The first derivative of $f(\theta)$ in Equation 48 with respect to $\theta$ is

$$
f^{\prime}(\theta)=2 \operatorname{Re}\left\{\mathbf{d}^{H}(\theta) \mathbf{Z}_{e}^{H} \mathbf{D}_{y}^{H}(\phi) \mathbf{C}_{y}^{H} \widetilde{\mathbf{B}}^{H} \widetilde{\mathbf{E}}_{\mathrm{n}} \widetilde{\mathbf{E}}_{\mathbf{n}}^{H} \widetilde{\mathbf{B}} \mathbf{C}_{y} \mathbf{D}_{y}(\phi) \mathbf{Z}_{e} \mathbf{d}(\theta)\right\}
$$

And the second derivative of $f(\theta)$ is given by

$$
\begin{aligned}
& f^{\prime \prime}(\theta) \\
& =2 \mathbf{d}^{H}(\theta) \mathbf{Z}_{e}^{H} \mathbf{D}_{y}^{H}(\phi) \mathbf{C}_{\gamma}^{H} \mathbf{B}^{H} \widetilde{\mathbf{E}}_{\mathrm{n}} \widetilde{\mathbf{E}}_{\mathrm{n}}^{H} \mathbf{B C}_{\gamma} \mathbf{D}_{y}(\phi) \mathbf{Z}_{e} \mathbf{d}^{\prime}(\theta) \\
& +2 \operatorname{Re}\left\{\mathbf{d}^{H}(\theta) \mathbf{Z}_{e}^{H} \mathbf{D}_{\gamma}^{H}(\phi) \mathbf{C}_{\gamma}^{H} \mathbf{B}^{H} \widetilde{\mathbf{E}}_{\mathrm{n}} \widetilde{\mathbf{E}}_{\mathrm{n}}^{H} \mathbf{B C}_{\gamma} \mathbf{D}_{y}(\phi) \mathbf{Z}_{e} \mathbf{d}^{\prime \prime}(\theta)\right\}
\end{aligned}
$$

Combining (59)-(61), an expression of the bias for the elevation angle estimates at angle $\theta_{i}$ can be finally written as

$$
\hat{\theta}_{i}-\theta_{i} \approx-\frac{f^{\prime}\left(\theta_{i}\right)}{f^{\prime \prime}\left(\theta_{i}\right)}
$$

In order to verify the approximation (58) and (62), we perform $N_{e}=500$ independent experiments using a sparse UCA in Figure 2. In the simulation we used one source with SNR $=25 \mathrm{~dB}$ and $\varphi=200^{\circ}$ moving in the elevation range $\theta \in(0, \pi / 2)$ since both $\left\|\Delta \mathbf{a}_{\mathrm{b}}^{1}(\theta, \phi)\right\|$ and $\left\|\Delta \mathbf{a}_{b}^{2}(\theta, \phi)\right\|$ are highly dependent on the elevation angle. Figure $3 \mathrm{a}$ shows the estimated MSE and the approximated MSE computed from Equation 58 with different $M$ for the azimuth estimation. The approximated bias follows the trend of $\left\|\Delta \mathbf{a}_{b}^{1}(\theta, \phi)\right\|$. However, for the estimation, it works badly when the elevation angle is near $0^{\circ}$ and $90^{\circ}$. That is, because the estimate azimuth angles can be any value when the elevation angle is $0^{\circ}$ and $\left\|\Delta \widetilde{\mathbf{a}}_{\mathrm{b}}^{1}(\theta, \phi)\right\|$ is large when the elevation angle is near $90^{\circ}$. In fact, the bias computed from Equation 58 can be approximately expressed as a function of the truncation errors when there is only one source (see Appendix). Because the truncation errors are very small when the elevation angle is near $0^{\circ}$ (see Figure 2), the 


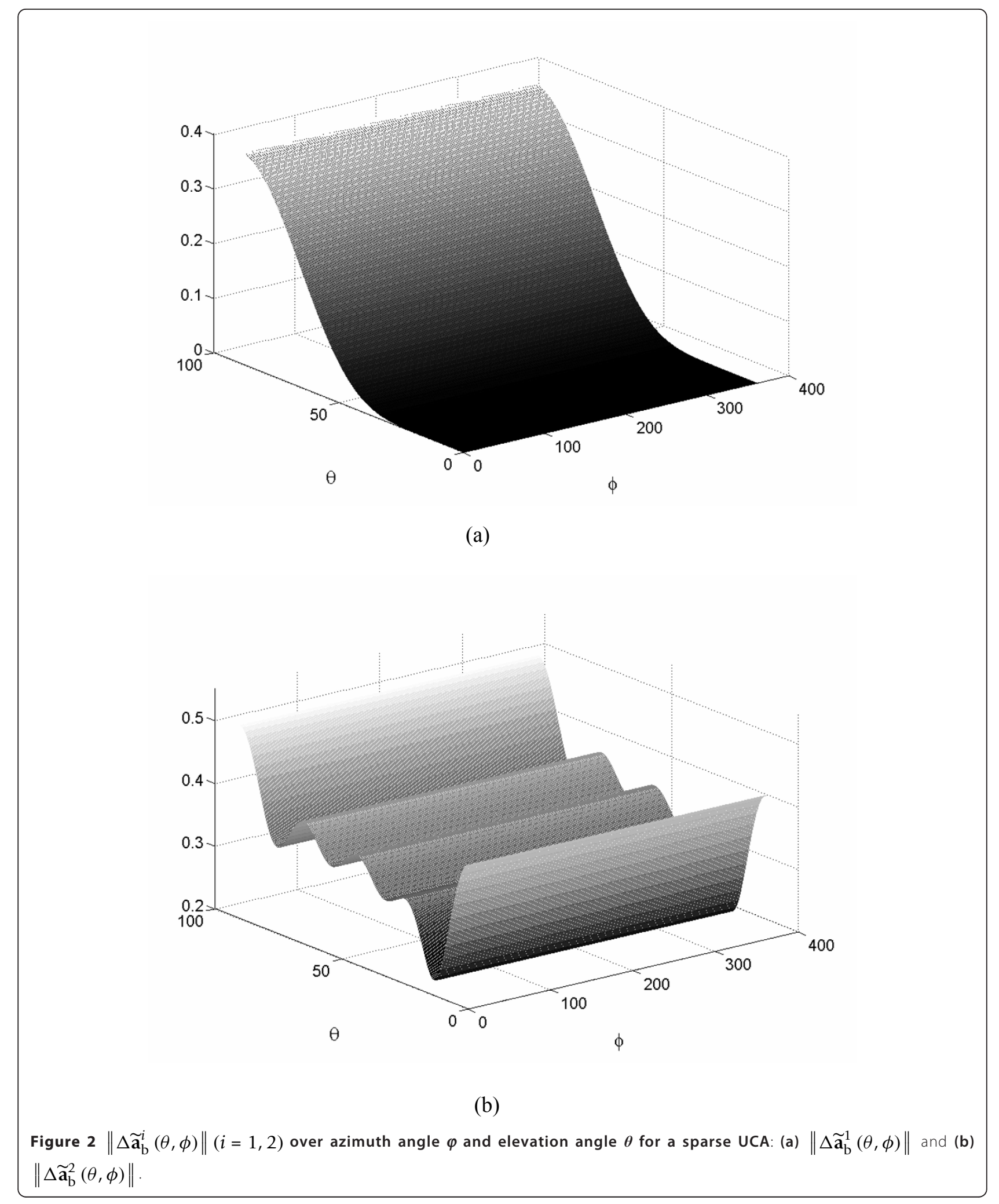




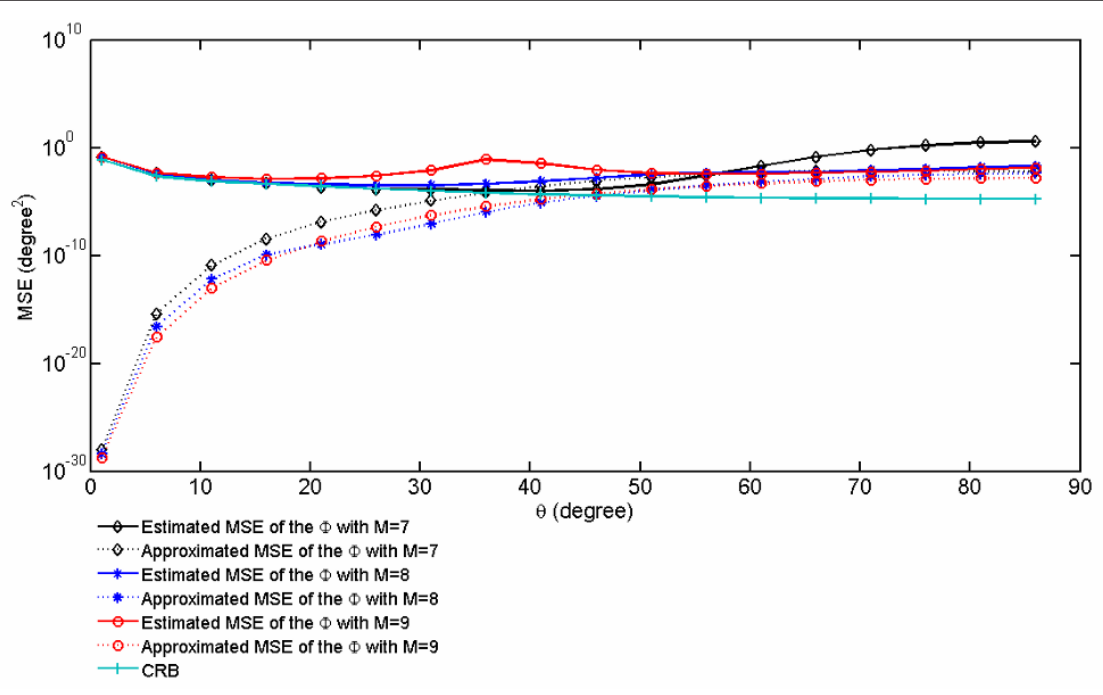

(a)

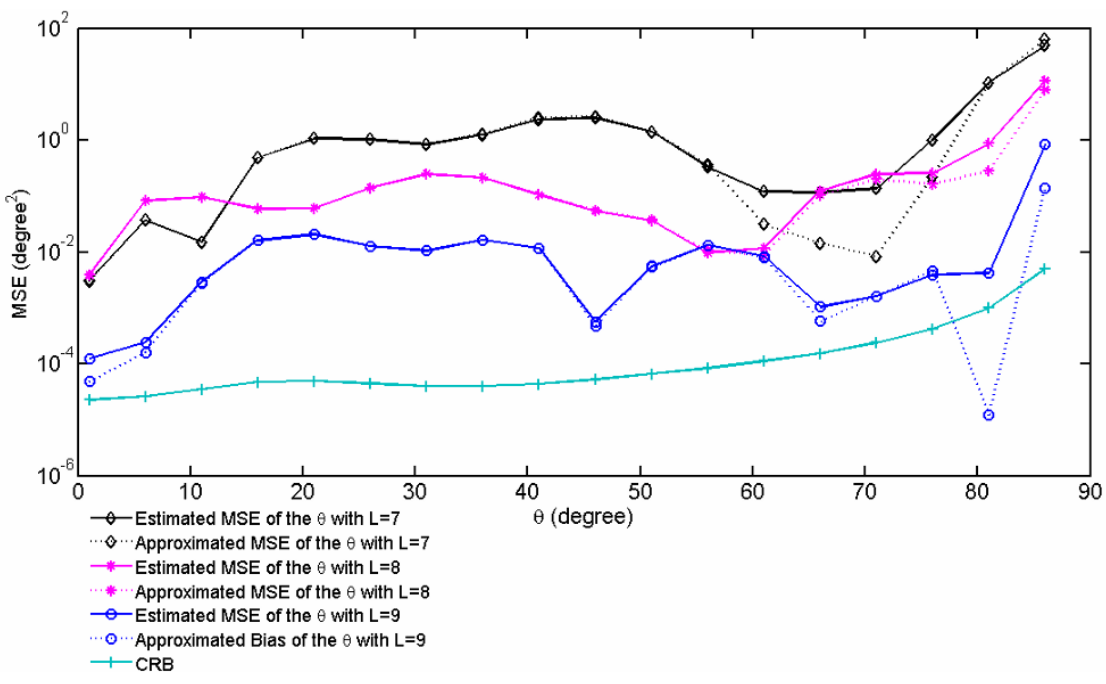

(b)

Figure 3 The estimated MSE and the approximated MSE for different truncated order and degree versus different elevation angle: (a) MSE and CRB for azimuth angle and (b) MSE and CRB for elevation angle.

approximated MSEs are small too. It seems like that the azimuth estimate accuracy does not increase when the truncated order $M$ increase in the whole elevation angle range. Hence, the truncated order $M$ can be $M=\lceil k r\rceil$. Figure 3b presents the estimated MSE and the approximated MSE computed from Equation (62) with different
$L$ for the elevation estimation. In this example, we only consider the influence of $\left\|\Delta \mathrm{a}_{\mathrm{b}}^{2}(\theta, \phi)\right\|$ on the estimate accuracy. Therefore, we use the real azimuth angle $\varphi=$ $200^{\circ}$ to estimate the elevation angle. It shows that the approximation calculated from Equation 62 describes the bias with high fidelity. And the elevation angle 
estimate accuracy is enhanced when $L$ increases. Hence, in order to obtain good estimate accuracy, a reasonable criterion to determine the truncated degree $L$ could be

$$
L=\min \left\{l \| l<2\lceil k r\rceil, \frac{\left|j_{l}(k r)\right|}{j_{-} \max }<\varepsilon\right\}
$$

where $j_{-} \max =\max \left\{\left|j_{l}(k r)\right|, l \leq 2\lceil k r\rceil\right\}$ and the predetermined $\varepsilon$ is related to the truncated accuracy.

\section{Simulations}

A sparse UCA of radius $r=\lambda$ with $N=11$ is employed in all experiments. The distance between each elements is $0.5635 \lambda$. The signals and noise in our simulations are assumed to be stationary, zero mean, and uncorrelated Gaussian random processes. Noise is both spatially and temporally white. The truncation order $M$ is 7 . In order to reduce the impact of the truncation errors on the estimate accuracy, the truncation degree here is $L=13$ for $\varepsilon=0.001$. The first example shows the spurious azimuth estimates of the original UCA-RARE. We consider the case of two impinging uncorrelated sources at $\left(\theta_{1}\right.$, $\left.\varphi_{1}\right)=\left(10^{\circ}, 40^{\circ}\right)$ and $\left(\theta_{2}, \varphi_{2}\right)=\left(30^{\circ}, 150^{\circ}\right)$. The SNR $=20$ $\mathrm{dB}$ is quoted per source per array element. Figure 4 depicts the root distributions nearby the unit circle for Equations 39 and 43. As shown in Figure 4a, except for the true azimuth roots $40^{\circ}$ and $150^{\circ}$ and their corresponding spurious roots $220^{\circ}$ and $330^{\circ}$, many other spurious roots introduced by the sparseness of the array element will appear if we employ the original UCARARE algorithm. Although the final estimates $(\theta, \varphi)$ may be obtained by calculating the MUSIC function, the computational cost due to solving the Root-MUSIC for every azimuth estimate will increase. Therefore, it is better to use the modified UCA-RARE (see Equation 43).

In the second example, one source with SNR $=25 \mathrm{~dB}$ and $\varphi=140^{\circ}$ is used to move in the elevation range $\theta \in$ $(0, \pi / 2)$. Two kinds of MCM, one obtained at $\theta=45^{\circ}$ and the other obtained at the accurate elevation angle, are employed to estimate the elevation angle. Sample statistics are computed from 500 independent trials. The root-mean-square-errors (RMSEs) and Cramer Rao Bounds (CRB) of the elevation estimate are shown in Figure 5. Although the result for the method with the MCM obtained at $\theta=45^{\circ}$ is a little inferior to the one with elevation-dependent MCM, the estimate difference for them is below $1^{\circ}$. It shows that it is feasible to get the elevation-dependent MCM by using the MCM obtained at $\theta=45^{\circ}$ first to obtain an initial estimate.

In the third example, the case of three impinging uncorrelated sources at $\left(\theta_{1}, \varphi_{1}\right)=\left(25^{\circ}, 60^{\circ}\right),\left(\theta_{2}, \varphi_{2}\right)=$ $\left(25^{\circ}, 120^{\circ}\right)$, and $\left(\theta_{3}, \varphi_{3}\right)=\left(50^{\circ}, 330^{\circ}\right)$ are considered. The $\mathrm{SNR}=20 \mathrm{~dB}$ is quoted per source per array element.
Figure 6a shows all azimuth roots distributions nearby the unit circle computed via Equation 43. Except the spurious azimuth estimates $60^{\circ}+180^{\circ}=240^{\circ}, 120^{\circ}+180^{\circ}$ $=300^{\circ}, 330^{\circ}-180^{\circ}=150^{\circ},\left(60^{\circ}+120^{\circ}\right) / 2=90^{\circ}$, and $90^{\circ}$ $+180^{\circ}=270^{\circ}$, we can find other spurious azimuth estimates caused by the sparseness of the array element in Figure $6 \mathrm{a}$. Figure $6 \mathrm{~b}$ depicts the corresponding elevation estimates distributions nearby the unit circle. It is easy to find that only the roots for the true azimuth estimates $\varphi_{i}$ are nearest by the circle and locate at $[0, \pi / 2]$. The elevation estimates roots for the spurious azimuth estimates $240^{\circ}, 300^{\circ}$, and $150^{\circ}$ are also very close to the circle, but locate at $[\pi, 2 \pi]$. The elevation estimates roots corresponding to the spurious azimuth estimates $90^{\circ}$ and $270^{\circ}$ are quite close to the unit circle and other spurious elevation estimates are all outside the unit circle. Hence, the spurious azimuth estimates $240^{\circ}, 300^{\circ}$, and $150^{\circ}$ can be eliminated automatically in the implementation of Root-MUSIC via judging their corresponding elevation estimates roots location. For the reserved paired DOAs $\left(\theta_{i}, \varphi_{i}\right)$ whose elevation roots locate at [0, $2 \pi$, besides to determine the final DOAs via computing the MUSIC function, here another approach is provided via judging the distance of elevation roots from the unit circle. We can consider the estimates corresponding to $D$ roots located closest to the unit circle as the final estimates.

The fourth example shows the performance of the proposed algorithm and MUSIC algorithm for different SNR levels. Sample statistics are computed from 500 independent trials. Two signals arrive at the array from two equal power sources from directions $\left(\theta_{1}, \varphi_{1}\right)=\left(10^{\circ}\right.$, $\left.20^{\circ}\right)$ and $\left(\theta_{2}, \varphi_{2}\right)=\left(30^{\circ}, 60^{\circ}\right)$, respectively. The SNR level is varied from 0 to $35 \mathrm{~dB}$ and is quoted per source per array element. For MUSIC algorithm, the DOA estimates are obtained by searching the highest local peaks over the 2D MUSIC spectrum. While for the proposed algorithm, the DOA estimates are found by implementing two computationally efficient rooting algorithms (the modified UCA-RARE and the Root-MUSIC algorithm). Here, the search step for MUSIC algorithm in both elevation and azimuth direction is $0.01^{\circ}$. The RMSEs plots of estimated DOAs and CRBs are shown in Figure 7. It is observed that the RMSEs of the estimates for the proposed algorithm and MUSIC algorithm follow the trend of the corresponding CRBs. The average Matlab-runtime to estimate the DOAs by the proposed algorithm is $0.78 \mathrm{~s}$ (simulated on a $2.8-\mathrm{GHz}$ Intel Core i5 CPU and 2.99 G Ram), whereas the average Matlab-runtime to estimate the DOAs by the traditional search of the 2D MUSIC spectrum is $7.43 \mathrm{~s}$. However, the estimated results for the proposed algorithm are comparable to the ones for MUSIC algorithm. 


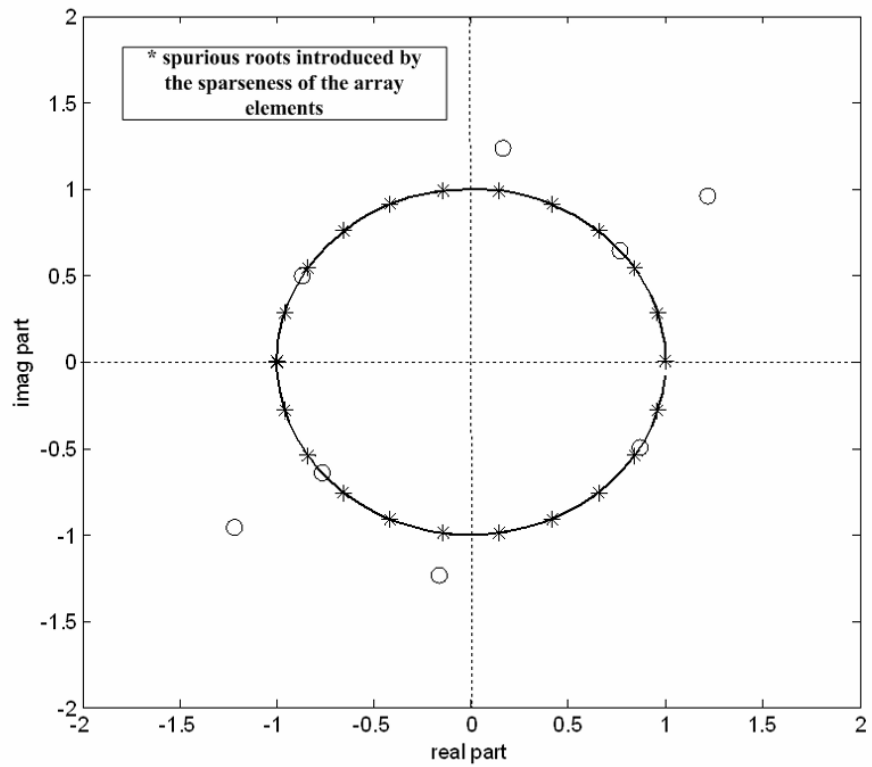

(a)

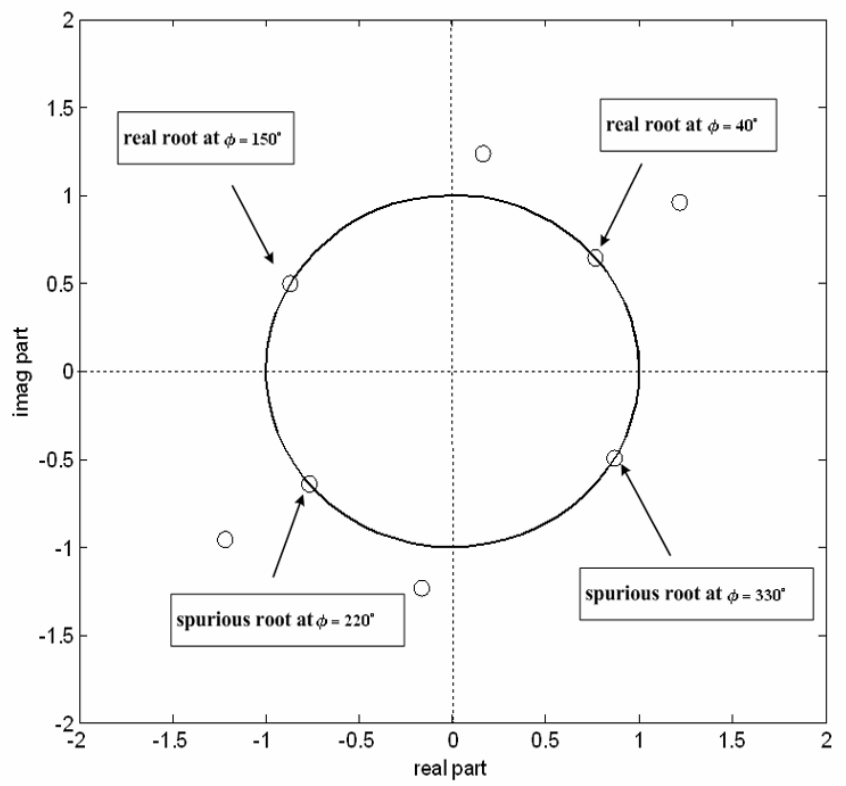

(b)

Figure 4 The azimuth estimated roots distribution nearby the unit circle: (a) roots for the original UCA-RARE and (b) roots for the modified UCA-RARE.

The last example is used to examine the capability of the proposed algorithm to estimate the DOAs of the closely spaced signals. Two uncorrelated sources with $\operatorname{DOAs}\left(\theta_{1}, \varphi_{1}\right)=\left(20^{\circ}, 100^{\circ}\right)$ and $\left(\theta_{2}, \varphi_{2}\right)=\left(20^{\circ}+\delta, 100^{\circ}\right.$ $+\delta$ ) are considered. The SNR $=25 \mathrm{~dB}$ is quoted per source per array element. Again the results are based on 500 independent trials. The DOA of the second source is varied as $\delta$ increases from $2^{\circ}$ to $30^{\circ}$. For each angle 


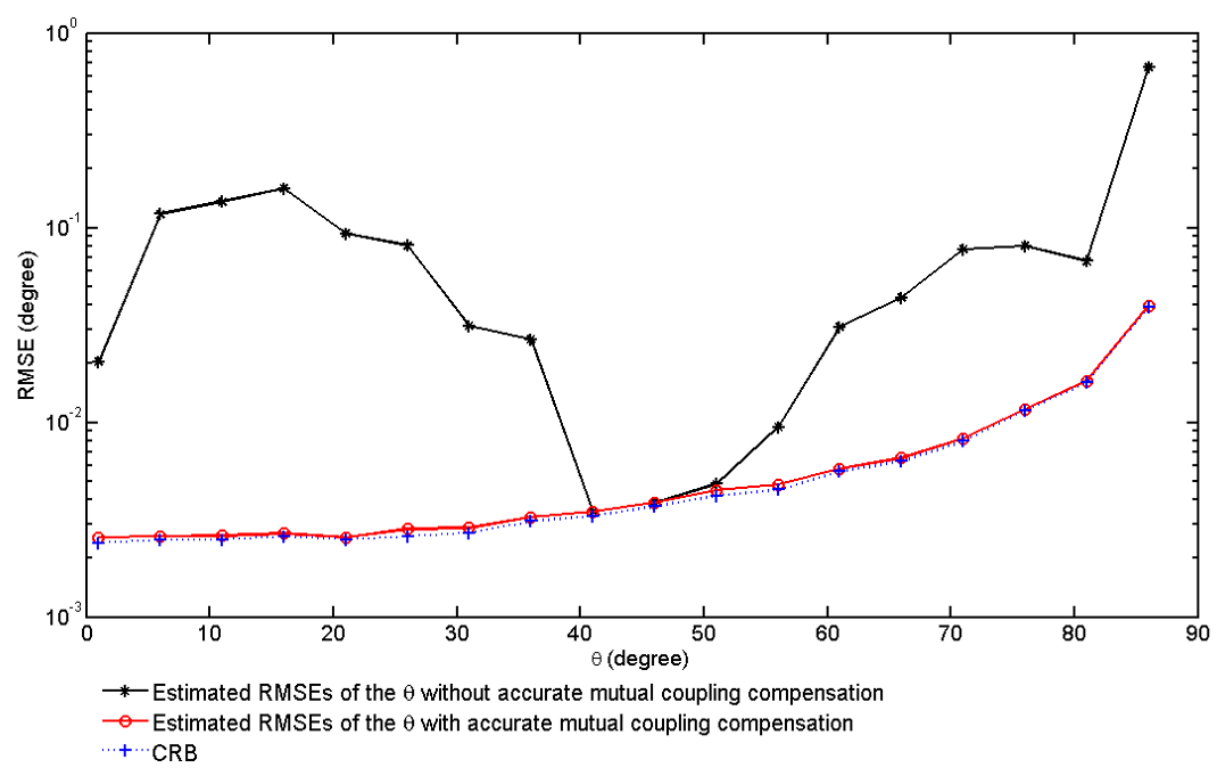

Figure 5 The RMSEs and CRB of elevation estimate versus different elevation angle.

separation the proposed and the MUSIC algorithms are applied to obtain the DOA estimates of the two impinging signals. The CRBs and RMSEs of the estimate values are shown in Figure 8. For smaller separation angles the accuracy decreases dramatically. The mean bias is smaller than $1^{\circ}$ for both two sources when separation angle is larger than $2^{\circ}$. Here, $L=13$ for $\varepsilon=0.001$ and the truncation error of the second kind of manifold decomposition can be nearly neglected for the proposed algorithm. The elevation angle estimation results for both algorithms are comparable. The truncation order is $M=$ 7 for the first kind of manifold decomposition. Its corresponding truncation error for the second source becomes larger along with the increase of the separation angle $\delta$ (see Section 5). Such truncation error could impact the DOA estimation accuracy for the proposed algorithm. Moreover, the azimuth estimates for the proposed algorithm are obtained without the exact knowledge of the mutual coupling. For MUSIC algorithm, there is no truncation error and the mutual coupling is compensated accurately. Hence, the azimuth angle estimation results for the proposed algorithm is a little inferior to the ones for MUSIC here.

\section{Conclusions}

Several algorithms for DOA estimation with UCAs are based on the traditional beamspace transform, which requires a sufficiently large number of elements to avoid aliasing in the steering vector of the mode space. Sometimes there may be a smaller number of antenna elements for application. We propose a new approach to estimate 2D DOAs for such UCAs. Two kinds of manifold decompositions are applied as the foundation of the proposed algorithm. In the first step, a modified sparse UCA-RARE is performed for the azimuth estimates. This step can be realized without the exact knowledge of elevation angle. It is proved by means of the Jacobi-Anger expansion (a decomposition of the element manifold into phase modes) that the sparse UCA-RARE is still applicable with a limited number of array elements. In the second step, the Root-MUSIC algorithm is used to obtain the elevation estimates via decomposing the manifold with Bauer's formula (an expansion of the array manifold into a double Fourier series). The influence of the truncation errors on the DOA estimate accuracy is analyzed and a method to choose the truncated degree for the elevation estimates is presented. Simulation results show that the proposed algorithm for sparse UCA can obtain good azimuth angle and elevation angle estimate results. The next challenge is to find a computational efficient method to handle the sparse UCAs with much wider inter-element spacing.

\section{Appendix}

Recalling Equation 42, the following equation holds true

$$
\mathbf{U}_{\mathrm{n}}^{H} \mathbf{T}^{H}\left(\phi_{i}\right) \mathbf{H}^{H} \widetilde{\mathbf{E}}_{\mathrm{n}} \widetilde{\mathbf{E}}_{\mathrm{n}}^{H} \mathbf{H T}\left(\phi_{i}\right) \mathbf{U}_{\mathrm{n}}=\mathbf{0}
$$

Let $\theta_{k}^{1}\left(k=1, \ldots, m_{i}\right)$ is the elevation angle of the $m_{i}$ sources with the same azimuth angle $\varphi_{i}$ and $\theta_{p}^{2}(p=1, \ldots$, $n_{i}$ ) is the elevation angle of the $n_{i}$ sources with the 


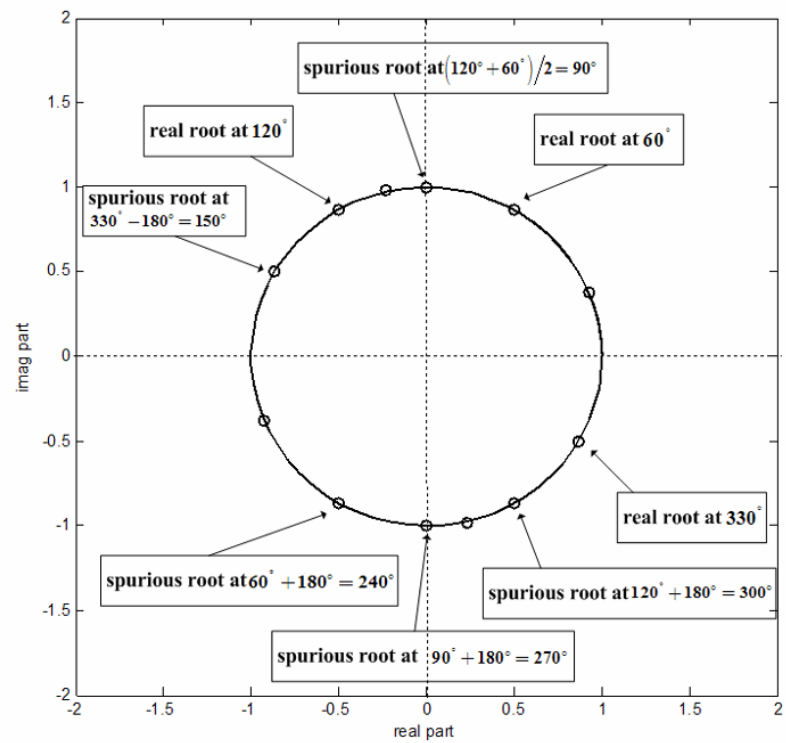

(a)

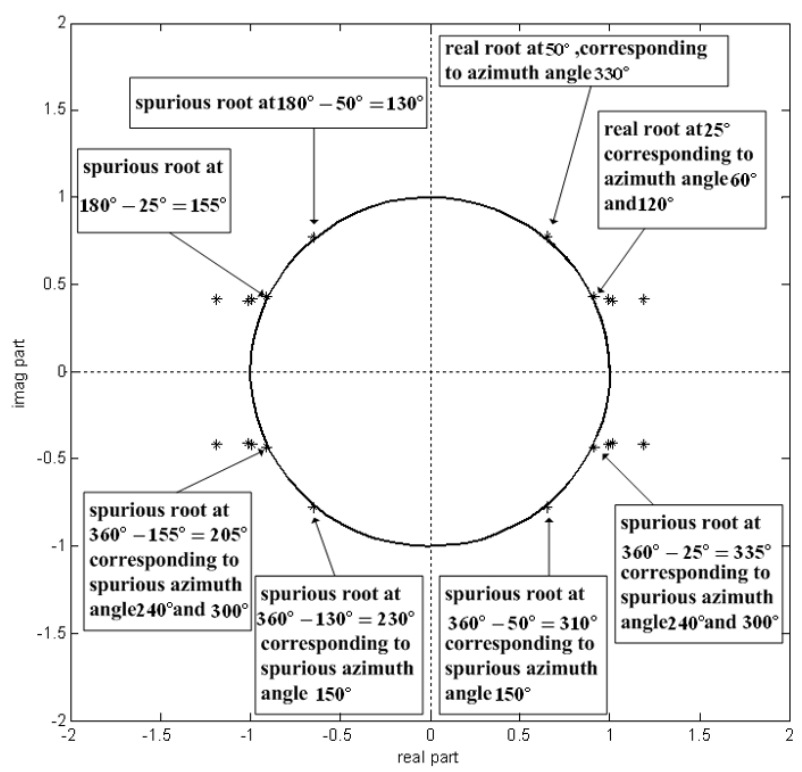

(b)

Figure 6 The estimated roots distribution for the proposed algorithm: (a) roots of azimuth estimates and (b) roots of elevation estimates. 


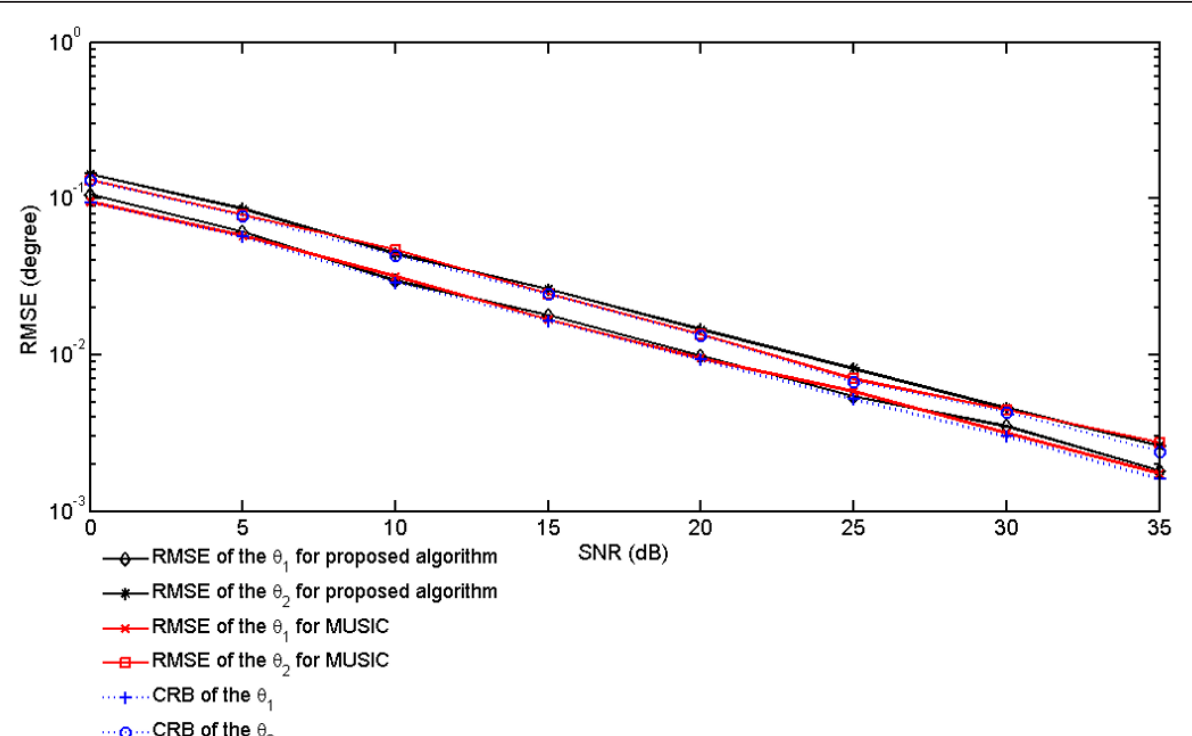

(a)

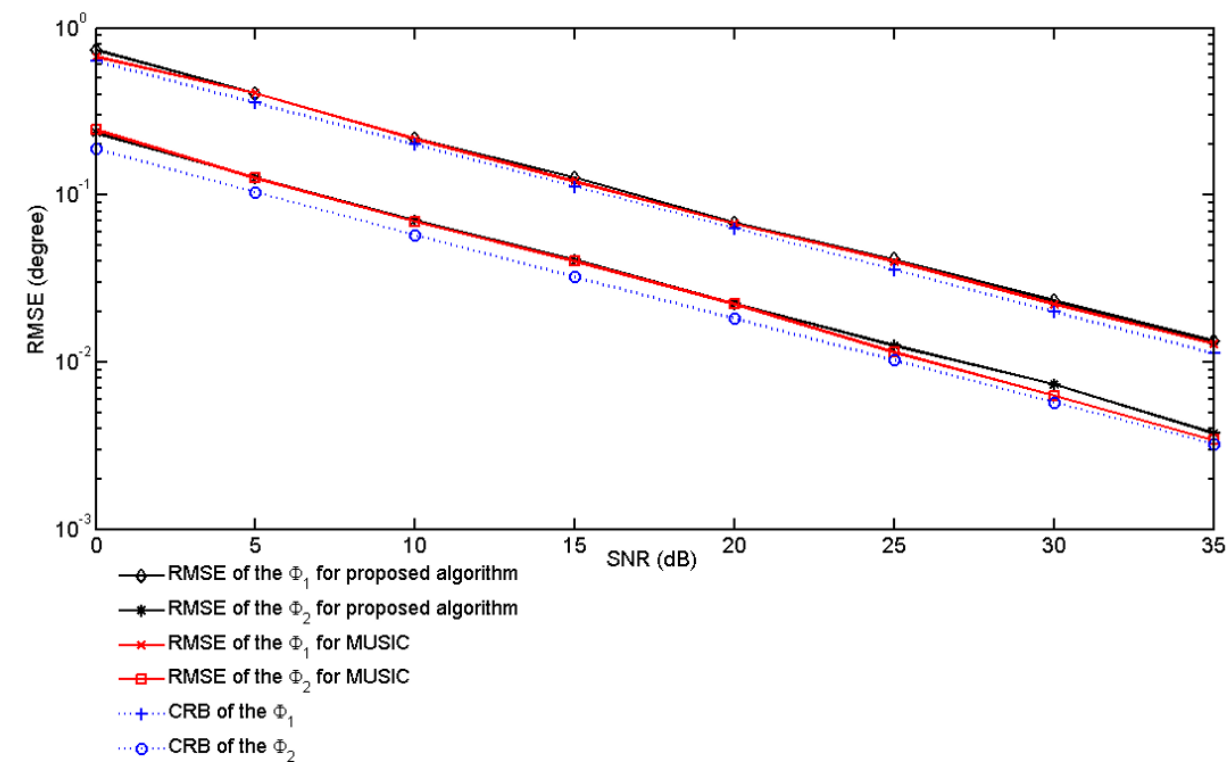

(b)

Figure 7 The RMSEs and CRBs of 2D DOA estimates versus SNR level: (a) RMSEs and CRB of the elevation estimates and (b) RMSEs and CRB of the azimuth estimates.

azimuth angle $\varphi_{i}+\pi$ or $\varphi_{i}-\pi$. There are

$$
\widetilde{\mathbf{g}}^{H}\left(\theta_{k}^{1}\right) \mathbf{T}^{H}\left(\phi_{i}\right) \mathbf{H}^{H} \widetilde{\mathbf{E}}_{\mathbf{n}} \widetilde{\mathbf{E}}_{\mathbf{n}}^{H} \mathbf{H T}\left(\phi_{i}\right) \widetilde{\mathbf{g}}\left(\theta_{k}^{1}\right)=0
$$

and

$$
\begin{aligned}
& \widetilde{\mathbf{g}}^{H}\left(\theta_{p}^{2}\right) \mathbf{T}^{H}\left(\phi_{i} \pm \pi\right) \mathbf{H}^{H} \widetilde{\mathbf{E}}_{\mathrm{n}} \widetilde{\mathbf{E}}_{\mathbf{n}}^{H} \mathbf{H T}\left(\phi_{i} \pm \pi\right) \widetilde{\mathbf{g}}\left(\theta_{p}^{2}\right) \\
& =\widetilde{\mathbf{g}}^{H}\left(\theta_{p}^{2}\right) \mathbf{D}_{\pi}^{H} \mathbf{T}^{H}\left(\phi_{i}\right) \mathbf{H}^{H} \widetilde{\mathbf{E}}_{\mathrm{n}} \widetilde{\mathbf{E}}_{\mathbf{n}}^{H} \mathbf{H T}\left(\phi_{i}\right) \mathbf{D}_{\pi} \widetilde{\mathbf{g}}\left(\theta_{p}^{2}\right)=0
\end{aligned}
$$

where $\quad \mathbf{D}_{\pi}=\operatorname{diag}\left(e^{j M \pi} e^{j(M-1) \pi} \cdots 1\right)$. Define

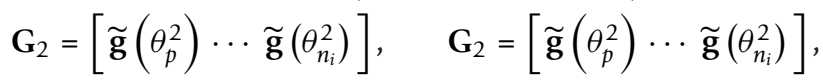
and $\mathbf{G}=\left[\begin{array}{ll}\mathbf{G}_{1} & \mathbf{D}_{\pi} \mathbf{G}_{2}\end{array}\right]$. With $\mathbf{G}$ and $\mathbf{U}_{\mathrm{n}}$ spanning the same subspace, there exists a full rank matrix $\mathbf{L} \in \mathbb{C}^{\left(m_{i}+n_{i}\right) \times\left(m_{i}+n_{i}\right)}$ allowing $\mathbf{U}_{\mathbf{n}}=\mathbf{G L}$. Hence, there is

$$
\mathbf{u}_{q}=\mathbf{G l}_{q}
$$




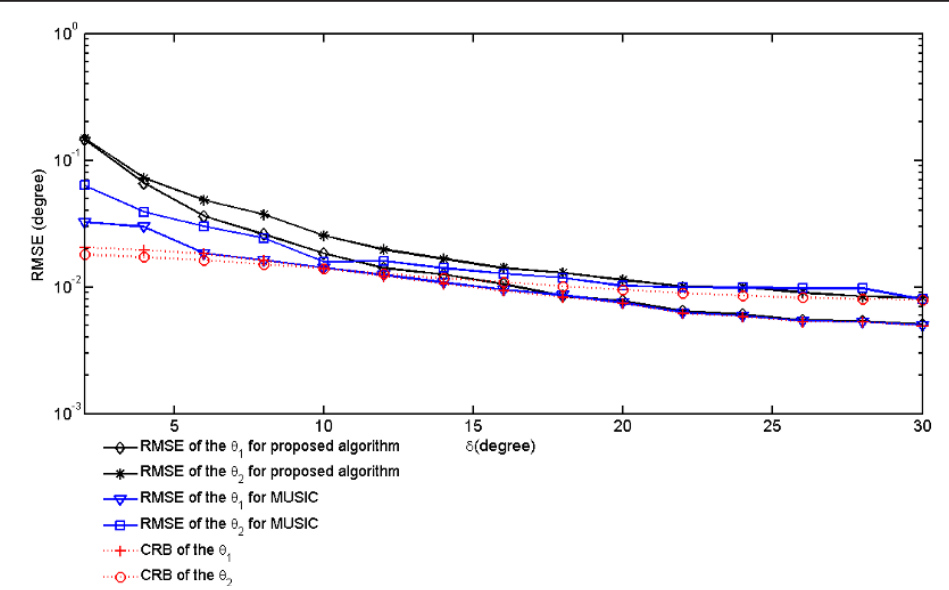

(a)

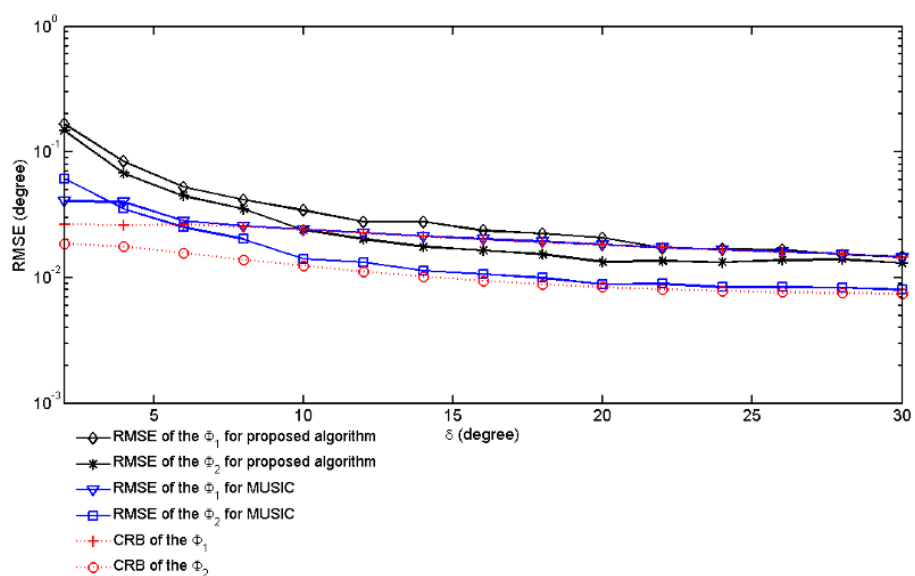

(b)

Figure 8 The RMSEs and CRBs of 2D DOA estimates versus the separation angle $\boldsymbol{\delta}$ : (a) RMSES and CRB of the elevation estimates and (b) RMSEs and CRB of the azimuth estimates.

where $\mathbf{I}_{q}$ represents the $q$ th columns of $\mathbf{L}$. Substituting Equation 67 in Equations 56 and 57, we get

$$
\gamma^{\prime}(\phi)=\sum_{q=1}^{m_{i}+n_{i}} 2 \operatorname{Re}\left\{\mathbf{l}_{q}^{H} \mathbf{G}^{H} \mathbf{T}^{H}(\phi) \mathbf{H}^{H} \widetilde{\mathbf{E}}_{\mathbf{n}} \widetilde{\mathbf{E}}_{\mathbf{n}}^{H} \mathbf{H T}(\phi) \mathbf{G l}_{q}\right\}
$$

and

$$
\begin{gathered}
\gamma^{\prime \prime}(\phi)=\frac{\partial y^{\prime}(\phi)}{\partial \phi} \\
=\sum_{q=1}^{m_{i}+n_{i}} 2 \mathbf{l}_{q}^{H} \mathbf{G}^{H} \mathbf{T}^{H}(\phi) \mathbf{H}^{H} \widetilde{\mathbf{E}}_{\mathrm{n}} \widetilde{\mathbf{E}}_{\mathbf{n}}^{H} \mathbf{H T}^{\prime}(\phi) \mathbf{G}_{q} \\
+\sum_{q=1}^{m_{i}+n_{i}} 2 \operatorname{Re}\left\{\mathbf{l}_{q}^{H} \mathbf{G}^{H} \mathbf{T}^{\prime \prime}(\phi) \mathbf{H}^{H} \widetilde{\mathbf{E}}_{\mathrm{n}} \widetilde{\mathbf{E}}_{\mathrm{n}}^{H} \mathbf{H T}(\phi) \mathbf{G}_{q}\right\}
\end{gathered}
$$

When $m_{i}+n_{i}=1$, Equations 68 and 69 can be expressed as

$$
\gamma^{\prime}(\phi)=2\left|l_{i}\right|^{2} \operatorname{Re}\left\{\widetilde{\mathbf{g}}(\theta)^{H} \mathbf{T}^{H}(\phi) \mathbf{H}^{H} \widetilde{\mathbf{E}_{\mathbf{n}}} \widetilde{\mathbf{E}}_{\mathbf{n}}^{H} \mathbf{H T}(\phi) \widetilde{\mathbf{g}}(\theta)\right\}
$$

and

$$
\gamma^{\prime \prime}(\phi)=\frac{\partial \gamma^{\prime}(\phi)}{\partial \phi}
$$

$$
\begin{gathered}
=2\left|l_{i}\right|^{2} \widetilde{\mathbf{g}}(\theta)^{H} \mathbf{T}^{H}(\phi) \mathbf{H}^{H} \widetilde{\mathbf{E}}_{\mathbf{n}} \widetilde{\mathbf{E}}_{\mathbf{n}}^{H} \mathbf{H} \mathbf{T}^{\prime}(\phi) \widetilde{\mathbf{g}}(\theta) \\
+2\left|l_{i}\right|^{2} \operatorname{Re}\left\{\widetilde{\mathbf{g}}(\theta)^{H} \mathbf{T}^{\prime \prime H}(\phi) \mathbf{H}^{H} \widetilde{\mathbf{E}}_{\mathbf{n}} \widetilde{\mathbf{E}}_{\mathbf{n}}^{H} \mathbf{H T}(\phi) \widetilde{\mathbf{g}}(\theta)\right\}
\end{gathered}
$$

Due to the truncation errors, there is $\widetilde{\mathbf{E}}_{\mathrm{n}} \widetilde{\mathbf{E}}_{\mathrm{n}}^{H} \widetilde{\mathbf{a}}_{\mathrm{b}}(\theta, \phi)=\widetilde{\mathbf{E}}_{\mathrm{n}} \widetilde{\mathbf{E}}_{\mathrm{n}}^{H}\left(\mathbf{H T}(\phi) \widetilde{\mathbf{g}}(\theta)+\Delta \widetilde{\mathbf{a}}_{\mathrm{b}}^{1}(\theta, \phi)\right)=0$.

Hence, $\widetilde{\mathbf{E}}_{\mathrm{n}} \widetilde{\mathbf{E}}_{\mathrm{n}}^{H} \mathbf{H T}(\phi) \widetilde{\mathbf{g}}(\theta)=-\widetilde{\mathbf{E}}_{\mathrm{n}} \widetilde{\mathbf{E}}_{\mathrm{n}}^{H} \Delta \widetilde{\mathbf{a}}_{\mathrm{b}}^{1}(\theta, \phi)$ and Equation 70 can be expressed as

$$
\gamma^{\prime}(\phi)=-2\left|l_{i}\right|^{2} \operatorname{Re}\left\{\widetilde{\mathbf{g}}(\theta)^{H} \mathbf{T}^{H}(\phi) \mathbf{H}^{H} \widetilde{\mathbf{E}}_{\mathrm{n}} \widetilde{\mathbf{E}}_{\mathbf{n}}^{H} \Delta \widetilde{\mathbf{a}}_{\mathrm{b}}^{1}(\theta, \phi)\right\}
$$


Because $\widetilde{\mathbf{E}}_{\mathrm{n}} \widetilde{\mathbf{E}}_{\mathrm{n}}^{H} \mathbf{H T}(\phi) \widetilde{\mathbf{g}}(\theta) \approx 0$, the first term in Equation 60 is far greater than the second term. Thus, Equation 58 can be approximately expressed as

$$
\hat{\phi}_{i}-\phi_{i} \approx-\frac{\operatorname{Re}\left\{\widetilde{\mathbf{g}}(\theta)^{H}{\mathbf{T}^{\prime}}^{H}(\phi) \mathbf{H}^{H} \widetilde{\mathbf{E}}_{\mathrm{n}} \widetilde{\mathbf{E}}_{\mathrm{n}}^{H} \Delta \widetilde{\mathbf{a}}_{\mathrm{b}}^{1}(\theta, \phi)\right\}}{\widetilde{\mathbf{g}}(\theta)^{H} \mathbf{T}^{H}(\phi) \mathbf{H}^{H} \widetilde{\mathbf{E}}_{\mathrm{n}} \widetilde{\mathbf{E}}_{\mathrm{n}}^{H} \mathbf{H} \mathbf{T}^{\prime}(\phi) \widetilde{\mathbf{g}}(\theta)}
$$

\section{Acknowledgements}

This work was supported by the University Basic Research Fund, P. R. China (nos. ZYGX2010J015 and ZYGX2009J015).

\section{Competing interests}

The authors declare that they have no competing interests.

Received: 15 August 2011 Accepted: 8 December 2011

Published: 8 December 2011

\section{References}

1. NJ Li, JF Gu, P Wei, 2-D DOA estimation via matrix partition and stacking technique. EURASIP J Adv Signal Process. 2009(53), 1-8 (2009)

2. MD Zoltowski, M Haardt, CP Mathews, Closed-form 2-D angle estimation with rectangular arrays in element space pr beamspace via unitary ESPRIT. IEEE Trans Signal Process. 44(1), 316-328 (1996)

3. CP Mathews, MD Zoltowski, Eigenstructure techniques for 2-D angle estimation with uniform circular arrays. IEEE Trans Signal Process. 42(9), 2395-2407 (1994). doi:10.1109/78.317861

4. M Pesavento, JF Böhme, Direction of arrival estimation in uniform circular arrays composed of directional elements. in Proc Sensor Array and Multichannel Signal Processing Workshop 503-507 (2002)

5. R Goossens, H Rogier, A hybrid UCA-RARE/Root-MUSIC approach for 2-D direction of arrival estimation in uniform circular arrays in the presence of mutual coupling. IEEE Trans Antennas Propag. 55(3), 841-849 (2007)

6. TT Zhang, YL Lu, HT Hui, Compensation for the mutual coupling effect in uniform circular arrays for 2D DOA estimations employing the maximum likelihood technique. IEEE Trans Aerosp Electron Syst. 44(3), 1215-1221 (2008)

7. W Buhong, H Hontat, L Mookseng, Decoupled 2D direction of arrival estimation using compact uniform circular arrays in the presence of elevation-dependent mutual coupling. IEEE Trans Antennas Propag. 58(3), 747-755 (2010)

8. R Goossens, H Rogier, S Werbrouck, UCA Root-MUSIC with sparse uniform circular arrays. IEEE Trans Signal Process. 56(8), 4095-4099 (2008)

9. HT Hui, Improved compensation for the mutual coupling effect in a dipole array for direction finding. IEEE Trans Antennas Propag. 51(9), 2498-2503 (2003). doi:10.1109/TAP.2003.816303

10. MA Doron, E Doron, Wavefield modeling and array processing-Part I: spatial sampling. IEEE Trans Signal Process. 42(10), 2549-2559 (1994). doi:10.1109/ 78.324722

11. C Mario, R Andreas, K Visa, Unified array manifold decomposition based on spherical harmonics and 2-D Fourier basis. IEEE Trans Signal Process. 58(9), 4634-4645 (2010)

12. EW Weisstein, Jacobi-Anger expansion. Wolfram MathWorld, http:// mathworld.wolfram.com/Jacobi-AngerExpansion.html

13. EDE Witte, H Griffith, P Brennan, Phase mode processing for spherical arrays. Electron Lett. 39, 1430-1431 (2003). doi:10.1049/el:20030922

14. MA Doron, E Doron, Wavefield modeling and array processing-Part II: algorithms. IEEE Trans Signal Process. 42(10), 2560-2570 (1994). doi:10.1109/ 78.324723

15. MA Doron, E Doron, Wavefield modeling and array processing-Part III: resolution capacity. IEEE Trans Signal Process. 42(10), 2571-2580 (1994). doi:10.1109/78.324724

16. F Belloni, A Richter, V Koivunen, Performance of root-MUSIC algorithm using real-world arrays. in 14th Eur Signal Processing Conf (EUSIPCO), Florence, Italy 4-8 (2006)
17. F Belloni, A Richter, V Koivunen, Extension of root-MUSIC to non-ULA array configurations. in Presented at IEEE Int Conf Acoustics, Speech, Signal Processing (ICASSP), France 14-19 (2006)

18. F Belloni, A Richter, $V$ Koivunen, DoA estimation via manifold separation for arbitrary array structures. IEEE Trans Signal Process. 55(10), 4800-4810 (2007)

19. F Belloni, A Richter, $V$ Koivunen, Beamspace transform for UCA: error analysis and bias reduction. IEEE Trans Signal Process. 54(8), 3078-3089 (2006)

20. B Friedlander, AJ Weiss, Direction finding in the presence of mutual coupling. IEEE Trans Antennas Propag. 39(3), 273-284 (1991). doi:10.1109/ 8.76322

21. T Rahim, DEN Davies, Effect of directional elements on the directional response of circular antenna arrays. Microwaves Opt Antennas IEE Proc $\mathrm{H}$. 129(1), 18-22 (1982). doi:10.1049/ip-h-1.1982.0004

22. W Mati, S Jacob, Direction finding of coherent signals via spatial smoothing for uniform circular arrays. IEEE Trans Antennas Propag. 42(5), 613-620 (1994). doi:10.1109/8.299559

23. AL Swindlehurst, T Kailath, A performance analysis of subspace-based methods in the presence of model errors-l: the MUSIC algorithm. IEEE Trans Signal Process. 40(7), 1758-1774 (1992). doi:10.1109/78.143447

doi:10.1186/1687-6180-2011-127

Cite this article as: Xie et al:: 2D DOA estimation with sparse uniform circular arrays in the presence of mutual coupling. EURASIP Journal on Advances in Signal Processing 2011 2011:127.

\section{Submit your manuscript to a SpringerOpen ${ }^{\mathcal{O}}$ journal and benefit from:}

- Convenient online submission

- Rigorous peer review

- Immediate publication on acceptance

- Open access: articles freely available online

- High visibility within the field

- Retaining the copyright to your article

Submit your next manuscript at $\boldsymbol{~ s p r i n g e r o p e n . c o m ~}$ 